\title{
The Benefits of Psychosocial Interventions for Mental Health in People Living with HIV: A Systematic Review and Meta-analysis
}

\author{
Sanne van Luenen ${ }^{1}$ (D) Nadia Garnefski ${ }^{1} \cdot$ Philip Spinhoven $^{1,2} \cdot$ Pascalle Spaan $^{1} \cdot$ \\ Elise Dusseldorp $^{3} \cdot$ Vivian Kraaij $^{1}$
}

Published online: 30 March 2017

(c) The Author(s) 2017. This article is an open access publication

\begin{abstract}
In this systematic review and meta-analysis we investigated the effectiveness of different psychosocial treatments for people living with HIV (PLWH) and mental health problems. Additionally, characteristics that may influence the effectiveness of a treatment (e.g., treatment duration) were studied. PubMed, PsycINFO and Embase were searched for randomized controlled trials on psychosocial interventions for PLWH. Depression, anxiety, quality of life, and psychological well-being were investigated as treatment outcome measures. Sixty-two studies were included in the meta-analysis. It was found that psychosocial interventions for PLWH had a small positive effect on mental health $(\hat{g}=0.19,95 \%$ CI $[0.13,0.25])$. Furthermore, there was evidence for publication bias. Six characteristics influenced the effectiveness of a treatment for depression. For example, larger effects were found for studies with psychologists as treatment providers. To conclude, this systematic review and meta-analysis suggests that psychosocial interventions have a beneficial effect for PLWH with mental health problems.
\end{abstract}

Resumen En esta revisión sistemática y meta-análisis se ha investigado la efectividad de los diferentes tratamientos

Sanne van Luenen

S.van.luenen@fsw.leidenuniv.nl

1 Section of Clinical Psychology, Institute of Psychology, Faculty of Social and Behavioural Sciences, Leiden University, P.O. Box 9555, 2300 RB Leiden, The Netherlands

2 Department of Psychiatry, Leiden University Medical Center, Leiden, The Netherlands

3 Section of Methodology and Statistics, Institute of Psychology, Leiden University, Leiden, The Netherlands psicosociales para las personas viviendo con VIH (PVVIH) y con problemas de salud mental. Adicionalmente, hemos estudiado las características que pueden influir en la efectividad de un tratamiento (por ejemplo, duración del tratamiento). Como medidas de tratamiento, pruebas controladas aleatorizadas de las intevenciones psicosociales para depresión, ansiedad, calidad de vida y bienestar psicológico fueron buscadas en PubMed, PsycINFO y Embase. Sesenta y dos estudios se han incluido en el metanálisis. Hemos encontrado que intervenciones psicosociales para las PVVIH tuvieron un menor efecto positivo en la salud mental ( $\hat{g}=0.19$, IC del 95\% [0.13, 0.25]). Además, hubo evidencia de sesgo en la publicación. Seis características influyeron la efectividad del tratamiento para la depresión. Por ejemplo, hemos encontrado efectos mayores en estudios con psicólogos como proveedores del tratamiento. Concluyendo, esta revisión sistemática y meta-análisis indica que las intervenciones psicosociales tienen un efecto beneficioso para gente con PVVIH y con problemas de salud mental.

Keywords HIV · Psychosocial intervention - Mental health $\cdot$ Depression $\cdot$ Meta-analysis

\section{Introduction}

In recent decades, due to the use of antiretroviral medication, HIV has become a chronic illness instead of a disease that rapidly leads to severe sickness and death. However, people living with HIV (PLWH) may still suffer from physical symptoms, such as pain and lack of energy [1]. In addition, mental health problems such as depression and anxiety are common among PLWH [2-4]. In PLWH, the prevalence rate of mood disorders or clinically significant 
depressive symptoms is approximately $33 \%$ [5], and the prevalence rate of anxiety disorders about $20 \%$ [6]. Several psychosocial factors-such as isolation, stigma, discrimination, lack of support, and drug abuse-can contribute to feelings of depression and anxiety [4, 7]. In turn, mental health problems may have various negative consequences for PLWH. For example, it has been shown that PLWH with depressive symptoms have a higher risk of poor adherence to antiretroviral therapy (ART); they are also more at risk for HIV-related morbidity and mortality [8]. More specifically, in PLWH psychological factors (such as depressive symptoms and stress) have been shown to be related to immune measures, such as decreased cluster of differentiation 4 (CD4) cell count and increased viral load $[9,10]$. In view of the above research, it seems apparent that depression and anxiety in PLWH may decrease wellbeing and quality of life [11].

Given the impact that psychological symptoms have on the health and well-being of PLWH, it is very important to treat these symptoms. Several psychosocial interventions, such as cognitive behavioral therapy (CBT), supportive interventions, meditation, and stress management interventions, have been developed for PLWH with mental disorders. Various reviews and meta-analyses have found that these interventions are effective in reducing symptoms of depression, anxiety, and distress in PLWH [12-18]. The format of these interventions may differ from a groupbased or individualized format to interventions where the PLWH's partner or other family members are also involved. In addition to assessing the overall effectiveness of various psychosocial interventions, it is also important to compare interventions with each other and determine whether specific interventions stand out as the most effective to improve mental health in PLWH. With this information, more targeted treatment may be provided in the future. The interventions that seem to be the most effective could be offered first, which might improve the psychological care of PLWH. However, previous metaanalyses and reviews [12, 13, 15-18] have not compared interventions with each other to discover which specific psychosocial interventions are the most effective for PLWH with mental health problems.

Additionally, it is also meaningful to investigate whether certain characteristics may influence the effectiveness of the treatment. We can distinguish two types of characteristics that may act as moderators. The first type are characteristics of the intervention, such as treatment duration, intervention techniques, or the provider of the therapy. If we have more knowledge about the effect of treatment characteristics on the effectiveness of an intervention, this can be used to improve existing interventions by including the most beneficial aspects when designing new interventions for PLWH with mental health problems. If we find, for example, that interventions provided online or by a psychologist are more effective than interventions provided by others (e.g., peers), it may be useful to design new interventions that are provided online or by psychologists. The second type of possible moderators are characteristics of the study, such as the sample that was used or the type of control group. These characteristics may partly explain why some studies find larger effects than others. For instance, it may be that studies with many female participants show larger effects than studies with many males. If this is the case, it may be useful to consider this aspect in future studies. As yet, not much research is available about moderators of treatment effect in PLWH. However, moderating factors were taken into account in one meta-analysis, which found that stress-management interventions for PLWH reduced anxiety symptoms. In this research, the effect of the intervention was found to be larger when they included more women, more participants with anxiety symptoms at baseline, younger participants, and made less use of medication adherence information and/or planning in an intervention [16].

Most previous meta-analyses have focused on particular psychological interventions (e.g., CBT or meditation) and mostly also on a particular outcome (such as alleviation of depression or anxiety) [12-18]. However, no meta-analysis has yet investigated which psychosocial interventions are the most effective on psychological outcomes. The first aim of the present systematic review and meta-analysis was to investigate this. This meta-analysis included various psychosocial interventions for PLWH, including CBT, support interventions (e.g., peer support), interpersonal psychotherapy, stress management, mindfulness, coping improvement interventions, and family interventions. In addition, multiple outcomes were studied: depression, anxiety, quality of life, and psychological well-being. Only randomized controlled trials (RCTs) were included in the meta-analysis. As it is important to investigate moderators of intervention effect, and this was not examined in detail in previous meta-analyses, the second aim of the current meta-analysis was to carry out a moderator analysis. Since we included a lot of different intervention types and outcomes, we expected that there would be large differences between study effect sizes. A moderator analysis could give more information about which characteristics might explain these differences in results. Various possible moderators were included: intervention characteristics such as intervention duration, therapy provider, and intervention techniques (e.g., stress-management); and study characteristics such as attrition, study quality, and sample characteristics. To conclude: we investigated and provide an overview of the effectiveness for PLWH of psychosocial interventions in decreasing depression and anxiety and improving quality of life and psychological well-being. In 
addition, we investigated whether specific interventions stood out as having the greatest effect on these outcomes and we studied moderators of intervention effect.

\section{Methods}

\section{Search Strategy and Study Selection}

Several strategies were used to search for relevant RCTs. First, we searched in the electronic databases PubMed, PsycINFO, and Embase on September 29, 2014. Search words included terms related to HIV/AIDS, various types of psychosocial interventions (e.g., CBT, psychotherapy), and outcomes (e.g., depression, quality of life). The search strategy with keywords can be found in the Appendix. Second, we also searched for papers in the references of available meta-analyses and reviews about the subject.

Studies were included when they met all of the following criteria: (1) RCT; (2) evaluation of a psychosocial intervention (see definition below); (3) participants in the study are HIV positive and are 18 years or older; (4) year of data collection is later than 1995 (see explanation below); (5) the outcome variables that were studied belong to one or more of the following categories: depression, anxiety, psychological well-being, or quality of life; (6) studies were written in the English language; and (7) data to calculate effect sizes was present in the paper or retrieved from the authors. Regarding the second inclusion criterion, a psychosocial intervention was operationalized as an intervention that aimed to change thoughts, emotions and/or behavior of PLWH and had a psychosocial component. Therefore, physical interventions (such as exercise), were not included in the meta-analysis. Regarding the fourth inclusion criterion, we decided to include studies that collected data later than 1995, since antiretroviral medication was developed in 1996 and consequently the future prospects of PLWH changed a lot after that period. Furthermore, the outcome domains depression, anxiety and quality of life were specified a priori. Only the outcome psychological well-being was first intended for stress-related outcomes, but this was a rather small category, so we decided to enlarge it. Positive and negative affect, psychological functioning and general mental health were examples of concepts that were included in the outcome psychological well-being. Studies that had posttraumatic stress disorder (PTSD) as an outcome were not included in the meta-analysis. Regarding the seventh criterion, authors of the included papers were contacted to retrieve data that was not available in the paper. When the authors did not respond to the requests (even after reminder e-mails) and the data to calculate effect sizes was not available, the study was not included in the meta-analysis.
The first step was the selection of studies on title and abstract, and was performed by two persons (first and fourth author). The first 100 studies were selected by both authors independently, to determine the agreement among selectors, which was substantial [19], Cohen's kappa $=0.80$. Thereafter, both selected half of the remaining studies. For the second step of selecting studies, based on full text, the studies were divided among three persons (first and fourth author and a Master student in clinical psychology). The inclusion criteria, as described above, were used in the following order to ensure a fast and equal decision: $6,3,1,4,2,5,7$. When there was doubt about including a study, the paper was discussed with one or more of the other authors to make a decision.

\section{Problems of Multiplicity}

Some decisions had to be made when multiple papers were published about the same data or when multiple measuring instruments, time points or interventions were used in one study. When there were multiple papers about the same data, the paper with the most relevant outcome data was used as the main paper. Other papers were used to add information that was not present in the main paper. In addition, when there were multiple measuring instruments for one outcome, two instruments were included in the analysis and their data was averaged. The instruments that were most validated and comparable to other studies were chosen. When there were assessments at more than one time point after the termination of the intervention, the first time point (first post-test) was included in the main analysis. Moreover, we did investigate overall differences between time points post intervention. For this analysis, time points were classified into these categories: 0-3 months post intervention, more than 3-6 months post intervention, more than 6-9 months post intervention, and more than 9 months post intervention. When two assessments occurred in one time period, both were included in the analysis and the data was averaged. At last, when there were multiple intervention and/or control conditions in a study, they were all included in the analysis and coded as intervention or control conditions. In the analysis concerning the overall effectiveness of psychosocial interventions on mental health, the data of multiple intervention conditions was averaged. To investigate which interventions and techniques were most effective in the moderator analysis, all interventions were investigated separately. Therefore, some studies were represented multiple times in this analysis. To be included as an intervention condition, the intervention should have a psychosocial component. In the control condition, people were put on a waiting list, received standard care or were in an active control 
condition. This last category included for example (psycho)education, support and telephone check-ups.

\section{Data Extraction and Coding}

We developed a protocol to extract the data from the articles. The following information was extracted from the papers: year of publication, baseline scores on outcome variables, post-treatment results, follow-up results, country of data collection, years of recruitment, study setting (inpatient; outpatient; combination), number of participants in each group, percentage attrition, percentage females, mean age, percentage MSM, percentage participants with AIDS, mean number of years with HIV, percentage participants that use ART, screening on depression (yes; no), intervention type (symptom-oriented intervention; supportive intervention; meditation intervention), intervention techniques (relaxation; CBT; motivational interviewing; stressmanagement), intervention duration (total duration in hours; duration in weeks; number of sessions; duration of one session), therapy provider [psychologist/psychotherapist; counsellor (e.g., nurse, HIV specialist, social worker, trained facilitator); peer; none (e.g., computer); other], intervention format [group; individual; combination; other (e.g., family interventions)], primary focus of intervention (mental health; no mental health), primary outcome (mental health; no mental health), theory content of intervention (theory-driven; not theory-driven), type of control group (waiting list; standard care; active control group), length of follow-up, type of analysis [intent-to-treat (ITT); no ITT], and study quality (see next paragraph).

The intervention type variable included three categories: symptom-oriented interventions, supportive interventions and meditation interventions. These categories were created post hoc, after examining the content of the included interventions. The category symptom-oriented interventions included mostly cognitive and/or behavioral therapy, stress-management, and interpersonal therapy. Furthermore, the category supportive interventions consisted of (peer) support and psycho-education, and the category meditation interventions included interventions that incorporated mindfulness, meditation, or relaxation. Since the symptom-oriented interventions used various psychological techniques, it was further investigated whether symptom-oriented interventions that used a specific technique would have larger effects than symptom-oriented interventions that did not use this specific technique. This was investigated in the symptom-oriented interventions only, because the supportive and meditation interventions mostly did not make use of additional psychological techniques. The assessed intervention techniques were relaxation, CBT (defined as containing cognitive and/or behavioral techniques), motivational interviewing and stress-management.
One symptom-oriented intervention may use multiple psychological techniques. For example, a symptom-oriented intervention may include CBT techniques and relaxation techniques. The explanation of intervention types and intervention techniques is depicted in Table 1. The variable theory content of intervention contains two categories: (1) it was described that the intervention was based on theory or a theoretical model (e.g., social cognitive theory or the health belief model) or (2) it was not described that the intervention was based on theory or a theoretical model. The type of control group was categorized as waiting list, standard care or an active control condition. Standard care included for example standard medical care and referral to mental health services when needed. The last category included for instance (psycho)education, support and telephone check-ups.

The following information was asked from the authors, when it was not available in the paper: baseline, posttreatment and follow-up data, years of recruitment, number of participants in each group, percentage attrition, percentage females, mean age, intervention duration, therapy provider, intervention format, type of control group, length of follow-up and type of analysis. The data was extracted by two persons (first author and a psychologist). Both coded a portion of the studies and 17 of the studies (27\%) were coded by both authors. The intraclass correlation coefficient was calculated for the agreement on continuous variables, which was 0.99 . For the categorical variables a Cohen's kappa was calculated, this was 0.72 , which is substantial.

\section{Study Quality}

Study quality was assessed by using two methods: the Cochrane Collaboration's tool for assessing risk of bias [20] and three criteria from a review about defining empirically supported psychological treatments [21]. The Cochrane Collaboration's tool for assessing risk of bias consists of six domains. Four domains were used in this study: (1) sequence generation for allocation to conditions; (2) concealment of allocation to conditions; (3) addressing incomplete outcome data; and (4) selective outcome reporting. The domain blinding of participants and researchers was not used, because in almost all studies participants and researchers could not be blinded to the allocation to conditions. Furthermore, the domain other sources of bias was not used, because in most studies there were no other sources of bias. On each domain, a study received the judgement low risk of bias $(+)$, high risk of bias (-) or unclear risk of bias (?) using the criteria from the tool.

Furthermore, we used three criteria from a review about defining empirically supported psychological treatments, to 
Table 1 Intervention types and intervention techniques

\begin{tabular}{ll}
\hline Intervention types & $\begin{array}{l}\text { Intervention techniques (in symptom-oriented } \\
\text { interventions only) }\end{array}$ \\
\hline $\begin{array}{l}\text { Symptom-oriented intervention (e.g., cognitive and/or behavioral therapy, stress- } \\
\text { management, interpersonal therapy) }\end{array}$ & Relaxation \\
$\begin{array}{l}\text { Supportive intervention (e.g., support, psycho-education) } \\
\text { Meditation intervention (e.g., mindfulness, meditation, relaxation) }\end{array}$ & $\mathrm{CBT}$ \\
& $\begin{array}{l}\text { Motivational interviewing } \\
\text { Stress-management }\end{array}$ \\
\hline
\end{tabular}

assess the quality of administering the intervention: (1) the availability of a treatment manual that was followed (published or designed for the study); (2) the use of a training for the therapy providers (for the study or general training); and (3) treatment integrity was checked during the study (e.g., supervision of therapy providers, recording of sessions, checking of protocol adherence). For each criterion a study received a judgement of yes $(+$, low risk of bias), no ( - , high risk of bias), unclear (?) or not applicable (NA; e.g., when the intervention is a self-help program). Two persons (first author and a psychologist) rated the quality of the studies. Both rated a portion of the studies and 17 studies were rated by both authors to calculate their agreement. Cohen's kappa was 0.67 , which is substantial.

\section{Moderators}

The following moderators were investigated: country of data collection, first year of participant recruitment, percentage attrition, percentage females, mean age, percentage MSM, percentage participants with AIDS, mean number of years with HIV, percentage participants that use ART, screening on depression, intervention techniques, intervention duration, therapy provider, intervention format, primary focus intervention, primary outcome mental health, theory content of intervention, type of control group, type of analysis and study quality.

For the moderator analyses with continuous variables, the assumptions for meta-regression were checked (normality and linearity). None of the variables met both assumptions. Therefore, the continuous variables were transformed into categorical variables. The categorization was based on statistical and content related reasons. The variable first year of recruitment was categorized into three periods: 1996-2001, 2002-2006 and 2007-2012. The variable percentage of drop-out was separated into three categories: $0-10,10-20$ and $>20 \%$, as was the variable percentage of females: $0-20 \%, 20-80$ and $80-100 \%$. The variable mean age was divided based on a median split: $<42.40$ and $\geq 42.40$ years. The variable percentage MSM was divided into two categories: 0 and $>0 \%$ (because most studies had no MSM, so the median was $0 \%$ ). The variable percentage of people with AIDS was separated into two categories based on a median split: $<40$ and $\geq 40 \%$, as was the variable number of years with HIV: $<10.02$ and $\geq 10.02$ years and the variable percentage of people on ART: $<87$ and $\geq 87 \%$. Finally, the variable total intervention duration was divided into four categories: 1-5, 5-12, 12-18 and 18-30 h.

Study quality was included as a moderator in the analysis. When a study met $0-2$ out of seven quality criteria (0-2 times a +$)$, the study was classified as a study with low quality. When a study met 3-4 quality criteria, the study was classified as a study with medium quality and when 5-7 criteria were met, the study was classified as a study with high quality. A rating of unclear risk of bias was scored as a high risk of bias (-) in this classification. For studies with a judgement of NA on the three criteria regarding the quality of administering the intervention, a low quality rating was given to studies which had a low risk of bias rating on $0-1$ on the four other quality criteria, a medium quality rating was given to studies which had a low risk of bias on 2 of the other quality criteria and a high quality rating was for the studies which had a low risk of bias on $3-4$ of the other quality criteria.

\section{Data Analysis}

The program comprehensive meta-analysis (CMA; version 3 ) was used for the analysis. Hedges' $g$ was calculated as a measure of effect size. Baseline, post intervention and follow-up means, standard deviations, sample sizes and/or other available data were used to calculate effect sizes (e.g. $F, t$ or $p$ values). One study [22] reported median decreases in depression scores, instead of mean decreases. These medians were entered into CMA, because the means could not be retrieved. Also, five studies [23-27] found no differences between intervention and control conditions on one or more outcome measures, but no data was available. The effect sizes of these outcome measures of the studies were set at zero. Cohen's guidelines were applied to 
interpret effect sizes: 0.2 may indicate a small effect size, 0.5 may indicate a medium effect size and 0.8 may indicate a large effect size [28]. Two-tailed $p$-values were used in all analyses. In CMA, a correlation between pre- and posttest should be indicated for each study. Since this correlation was rarely reported in study papers, this was set at 0.5 (as suggested by [29]). Standardized residuals were inspected to find outliers, defined as studies with standardized residuals larger than $|3|[30]$.

A random effects model was used for the main analysis to estimate the pooled effect size of psychosocial interventions on mental health (expressed as Hedges' $\hat{g}$ ). Separate analyses were conducted for each outcome (depression, anxiety, quality of life, and psychological wellbeing), intervention type (symptom-oriented intervention, supportive intervention, and meditation intervention) and time point ( $0-3$ months post intervention, $3-6$ months post intervention, 6-9 months post intervention, and $>9$ months post intervention). The random effects model was used because we assumed heterogeneity across studies. To investigate the presence and amount of heterogeneity, $Q$ and $I^{2}$ were calculated. When $Q$ is significant, this means that the results of the studies are probably not consistent. The amount of heterogeneity can be identified with $I^{2}$. Values of $25 \%$ indicate low heterogeneity, $50 \%$ indicates moderate heterogeneity and $75 \%$ indicates high heterogeneity [31].

For the moderator analysis, a mixed effects model was used, in which the random effects model was used to combine studies in one subgroup and a fixed effects model was used to compare across subgroups [32]. In CMA, the mixed and random effects option was set to: do not assume a common among-study variance component across subgroups (do not pool within-group estimates of tau-squared).

To examine the presence of publication bias different methods were used. First, a funnel plot was created, where the standard error is plotted as a function of effect size. Studies with small standard errors (large studies in general) are displayed at the top of the plot and studies with large standard errors (small studies in general) are displayed at the bottom of the plot. When the studies are symmetrically distributed around the pooled effect size estimate, there is no indication of publication bias. When it seems that studies are missing on the lower left side, this may be an indication of publication bias (small studies with results lower than the pooled estimate are missing). Second, Egger's test of the intercept [33] was used to statistically test for publication bias. There is an indication of publication bias when the test is significant. Last, Duval and Tweedie's trim and fill analysis [34] was used to investigate whether it was necessary to impute studies in the funnel plot due to publication bias. After the imputation of missing studies, an adjusted effect size was calculated.

\section{Results}

Through electronic databases, 905 articles were identified (see flow-chart of study inclusion and exclusion in Fig. 1). After removal of duplicates (228), 677 articles were screened on title and abstract. Thereafter, 197 articles were screened on full text. After this screening, 64 studies met the inclusion criteria. In addition, three studies were found in previous meta-analyses and systematic reviews. From 20 of the 67 studies, data to calculate effect sizes was not present in the paper. Therefore, the authors were contacted to obtain these data. Of 15 studies, the authors were able to provide the data, one author could not provide the data, the authors of one study did not want to be included in the meta-analysis (because study aim did not fit with the aim of the meta-analysis), and three authors did not respond. In total, 62 studies were included in the meta-analysis.

\section{Study Characteristics}

The characteristics of all included studies are presented in Table 2. In total, 10,307 participants were randomized to intervention and control conditions (range 12-936; $k=62$ ). Drop-out (the percentage of participants that did not complete the first posttest) ranged from 0 to $55 \%$, with a mean of $18 \%(S D=11.93 ; k=58)$. Seven studies $(7 / 60)$ included only males and 13 included only females, the percentage of females in the included studies was $44 \%$ on average $(S D=34.54)$. The mean of the mean age of participants across studies was 42.01 years $(S D=5.98$, range of the mean: $26.00-59.00$ years, $k=54$ ). The mean percentage of MSM in the studies that reported on it was $31 \%$ $(S D=38.27 ; k=22)$. Across the studies that documented it, the mean percentage of people with AIDS was $45 \%$ $(S D=28.59 ; k=15)$ and the mean duration of HIV was 9.81 years $(S D=3.59$, range of the mean: $0-18$ years; $k=28$ ). Most participants in the studies that reported on it used ART, the mean percentage of people that used it is $76 \%(S D=34.28 ; k=28)$. For some characteristics, the number of studies that reported on it was very low. Therefore, these results should be interpreted with caution.

The majority of studies was conducted in the USA and Canada $(k=46)$. Other countries of data collection were China, Iran, Kenya, Nigeria, South Africa, Switzerland, Tanzania, Thailand, The Netherlands, Uganda, and Vietnam. One study recruited participants in the USA and Mexico and one study in South Africa, Puerto Rico, and the USA. The years of participant recruitment ranged from 1996 to $2013(k=57)$. The majority of studies was conducted in an outpatient setting (58/62), only two studies were conducted in an inpatient setting and two studies combined inpatients and outpatients. Ten studies (10/62) 


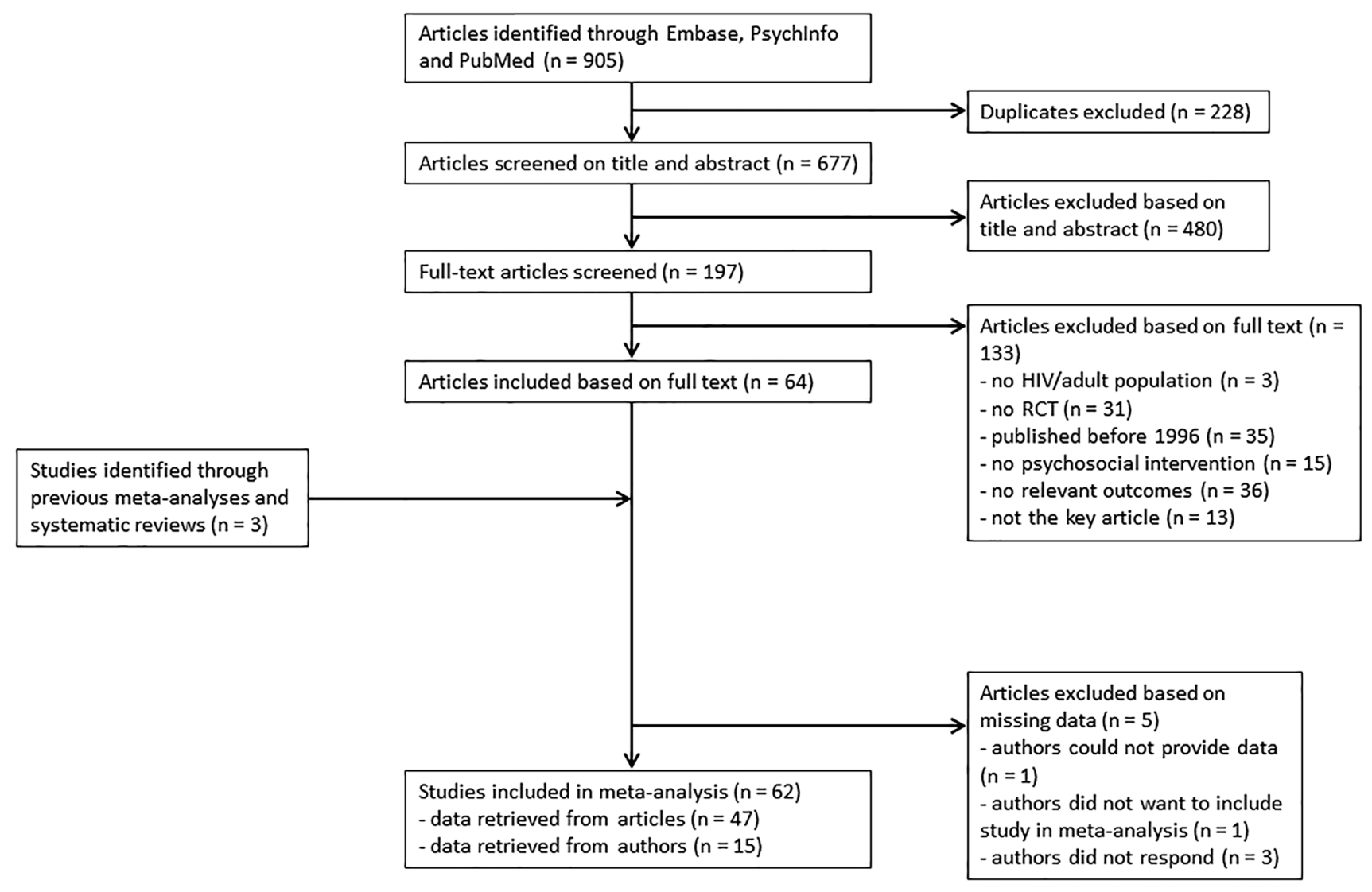

Fig. 1 Flow chart of study inclusion and exclusion

incorporated the presence of depressive symptoms as an inclusion criterion. In the majority of studies (54/62), mental health (i.e., depression, anxiety, quality of life, or psychological well-being) was a primary outcome measure. Depression was measured in 47 studies, anxiety in 22, quality of life in 19, and psychological well-being in 25 studies. Some studies (31/62) used an active control condition, 9 studies used a waiting list condition, and 22 studies had a standard care control condition. Furthermore, more than half of the studies (32/51) used an ITT analysis, and included one or more follow-up assessments (40/62); 22 studies had one follow-up, 16 studies had two followups, one study had 3 follow-ups, and one study had 4 follow-ups. The timing of follow-ups ranged from 1 to 17 months after treatment completion.

\section{Intervention Characteristics}

The description of intervention characteristics was based on all interventions, so eight studies [46, 52-54, 61, 64, 87, 91] were represented twice in this analysis and one study [65] was represented three times, because multiple interventions were investigated in these studies. The letter $\mathrm{m}$ will be used to indicate the number of interventions.
Regarding intervention types (see Table 1), a majority of the interventions were symptom-oriented (41/72), the rest were supportive (20/72), or meditation interventions (11/ 72). Regarding techniques used in symptom-oriented interventions ( $m=41$ ), CBT techniques were used in 29 interventions, relaxation techniques in 14, stress-management techniques in 16, and finally motivational interviewing techniques in 6 interventions. Almost two-thirds of the interventions (47/72) were focused on one of our outcome measures (depression, anxiety, quality of life, or psychological well-being). Studies that investigated interventions that were not focused on one of our outcome measures, were often aimed at medication adherence or sexual risk behavior, and sometimes at coping, disclosure, general health, family functioning, posttraumatic stress disorder symptoms, or smoking. A majority (44/72) of the interventions were theory-driven and 28 interventions were not theory-driven. Concerning the duration of the interventions, the total length ranged from 1 to $30 \mathrm{~h}(m=62)$, with a mean of $12.63(S D=8.46)$. The duration of the intervention in weeks ranged from 1 to $54(M=12.20$, $S D=13.27, m=66)$ and the number of sessions ranged from 1 to $48(M=9.92, S D=8.62, m=67)$. The average length of one session was $1.37 \mathrm{~h}(S D=0.66$, range $15 \mathrm{~min}$ 


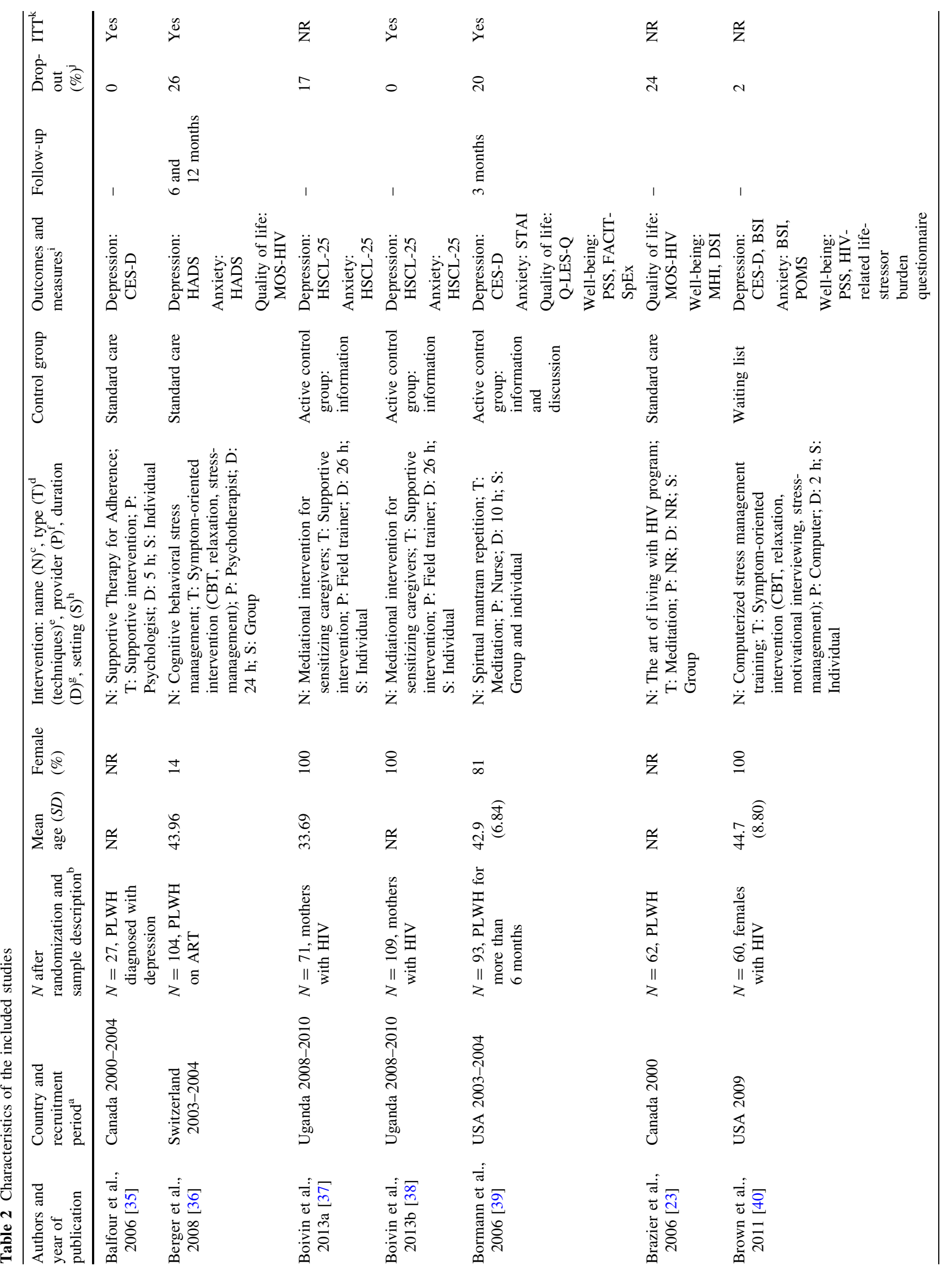




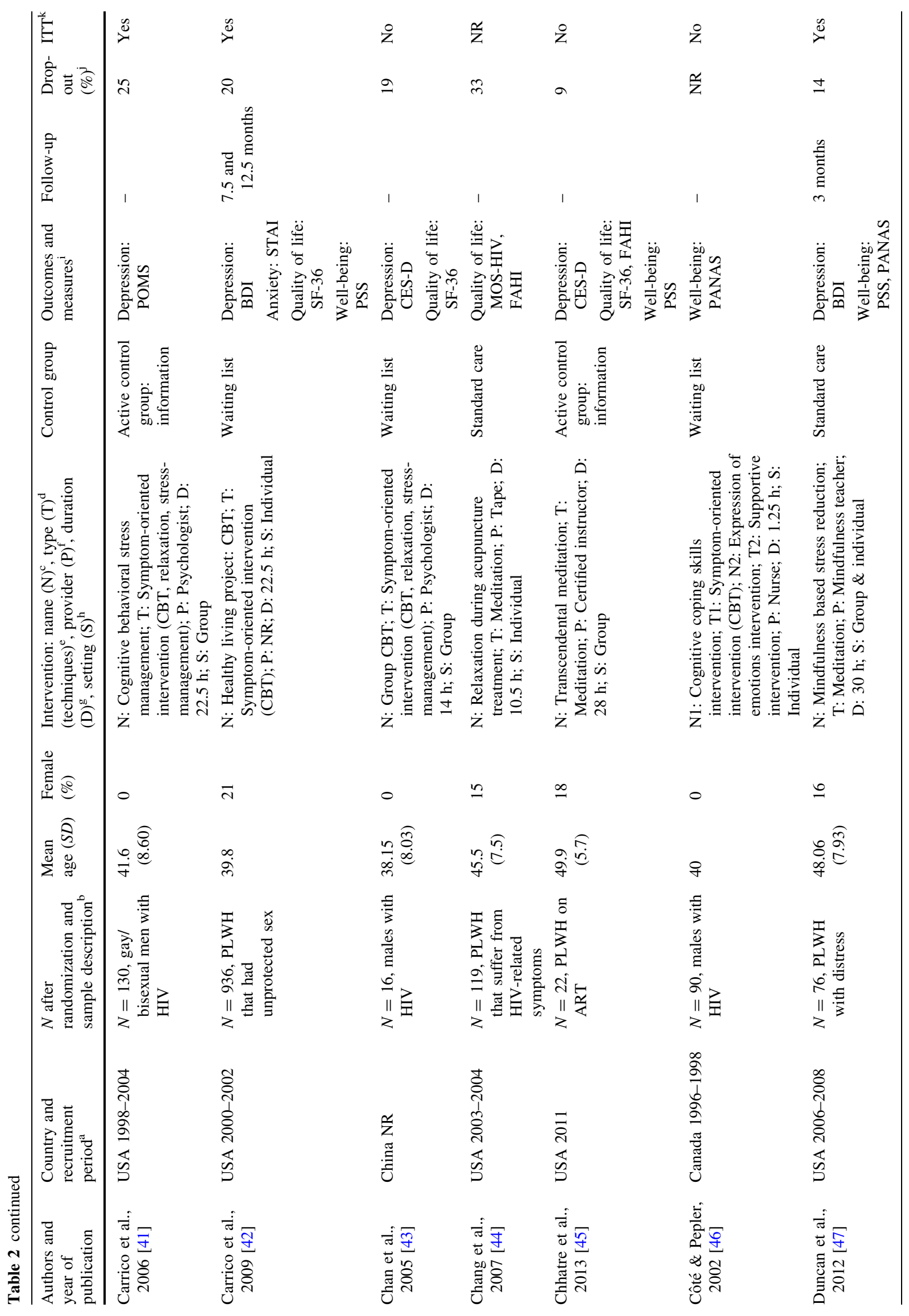




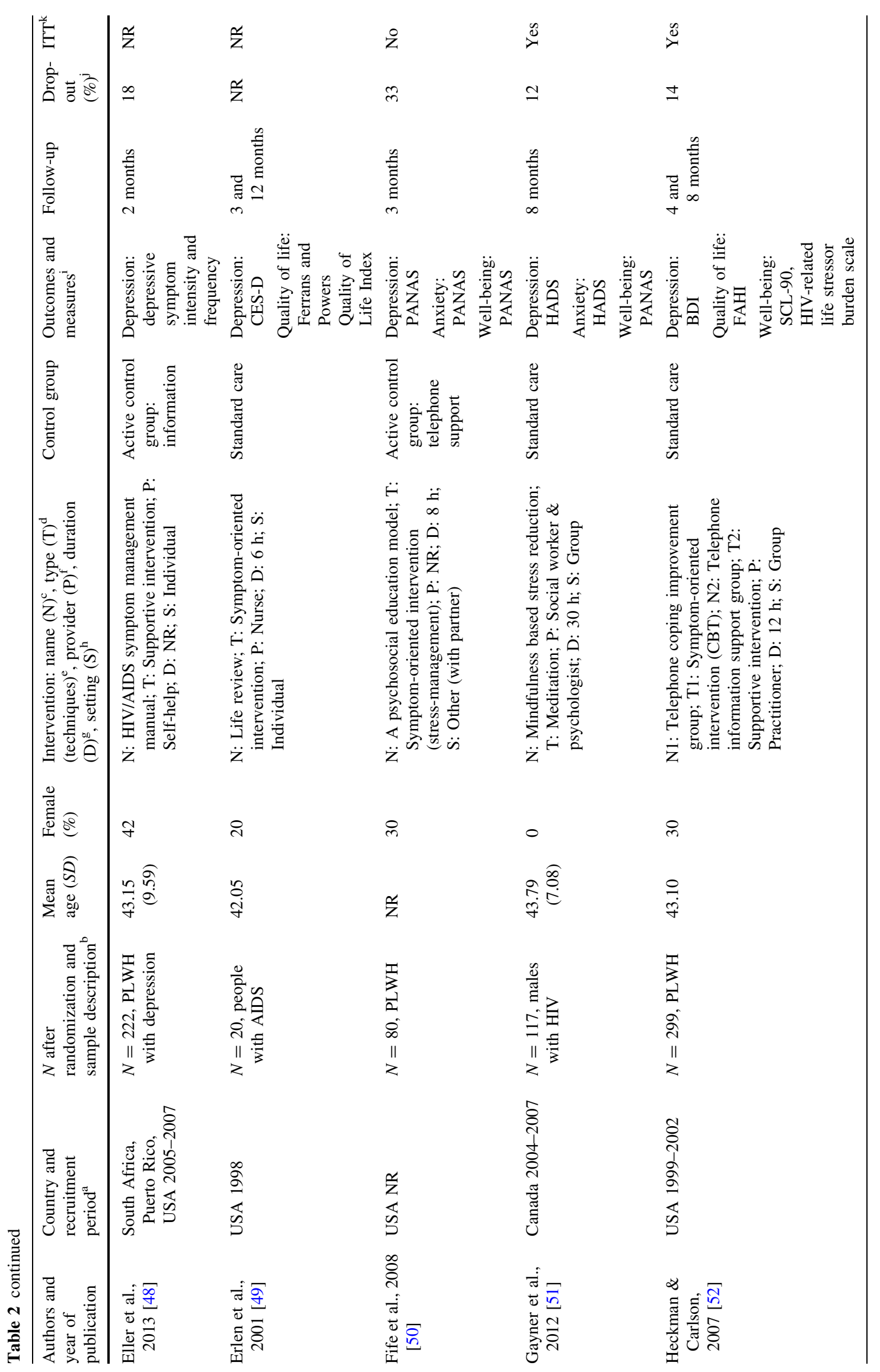




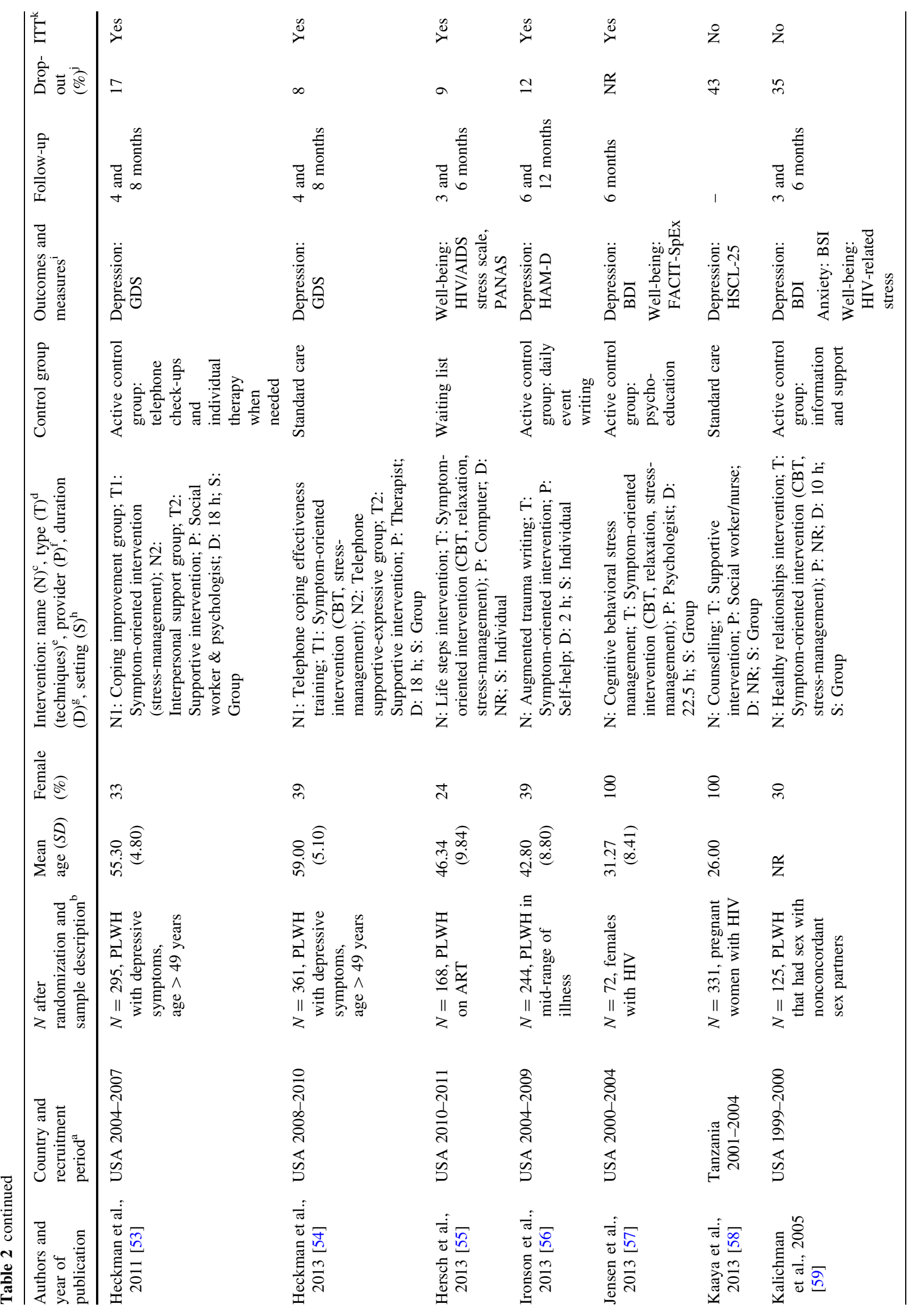




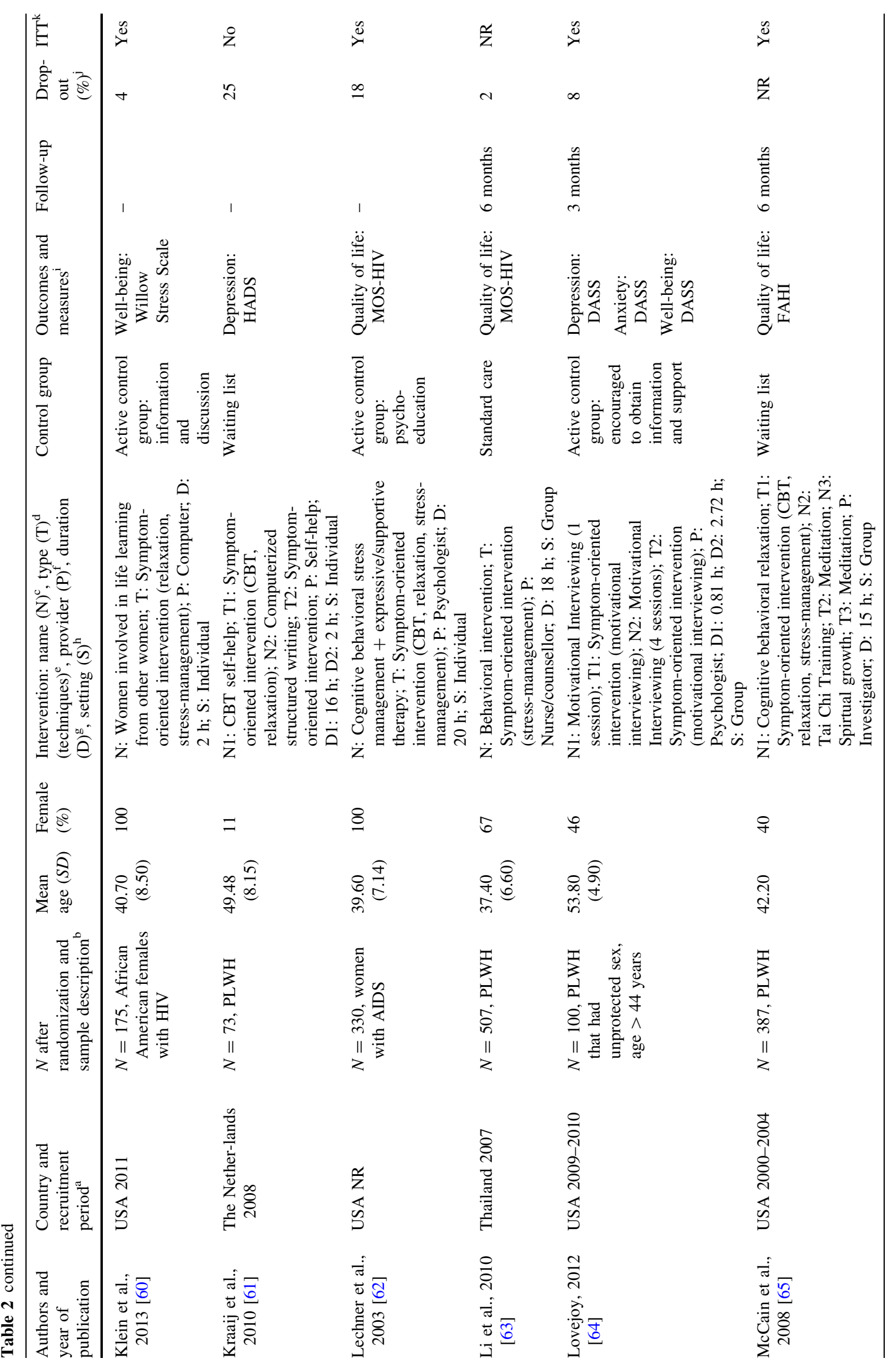




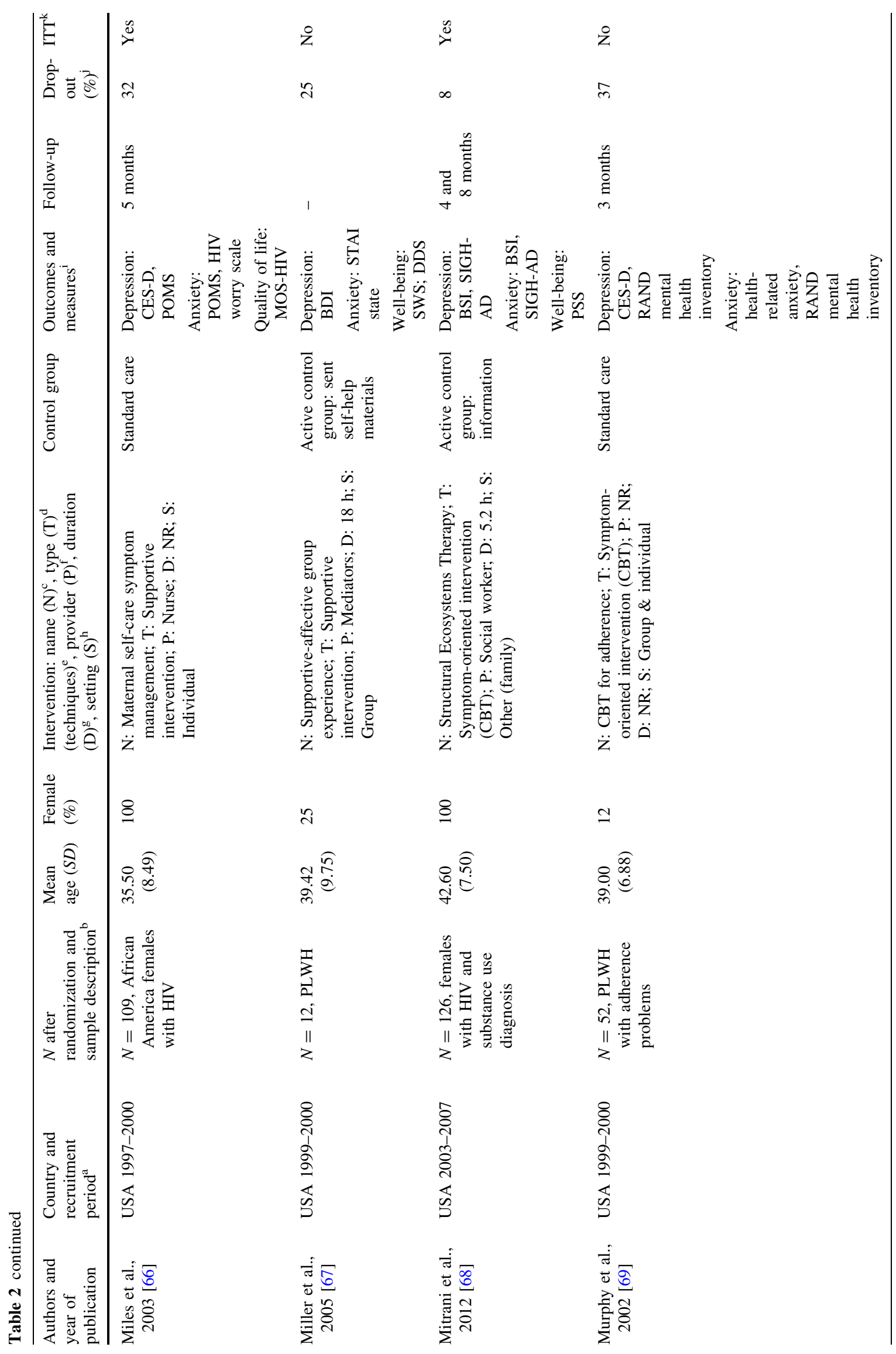




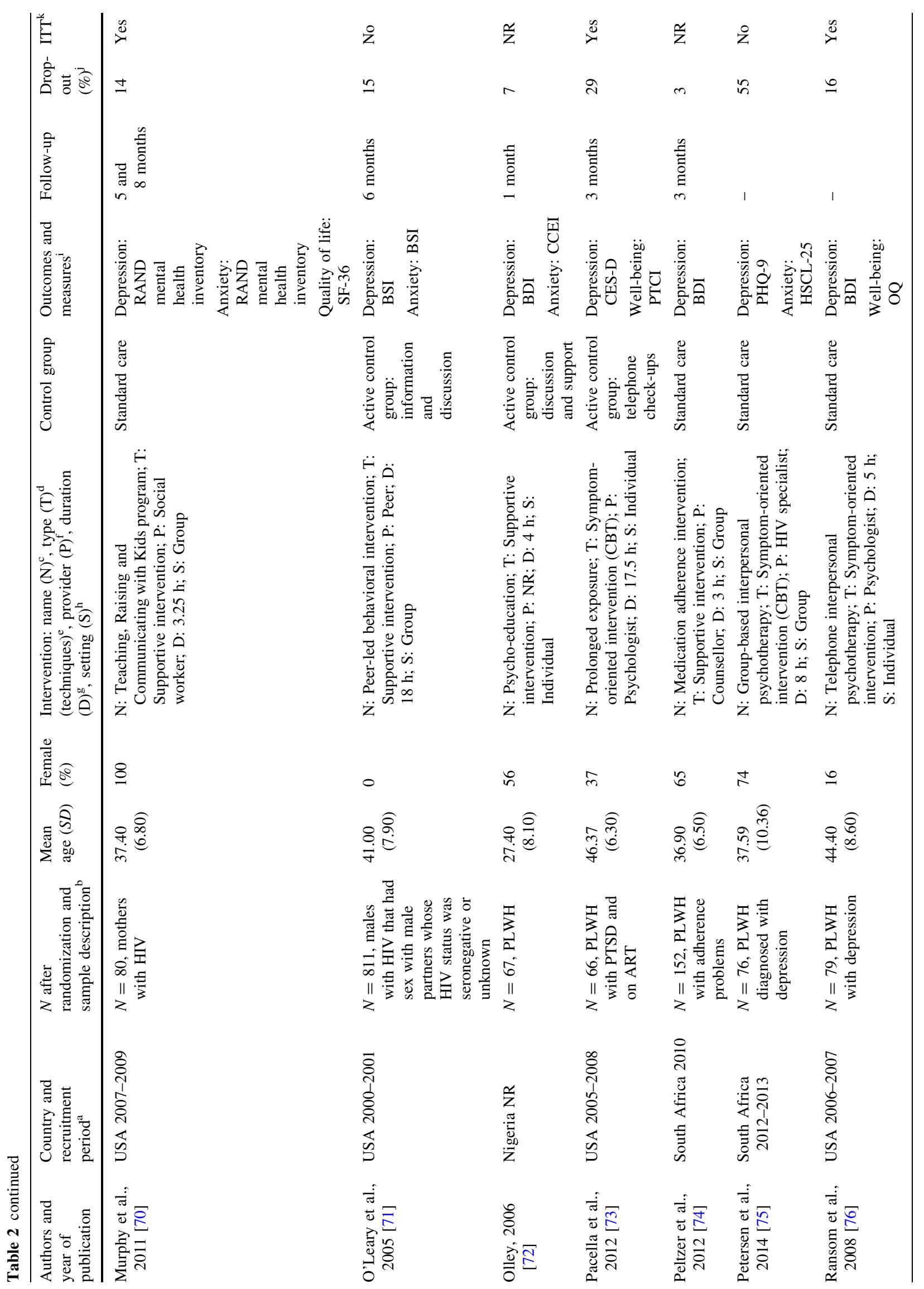




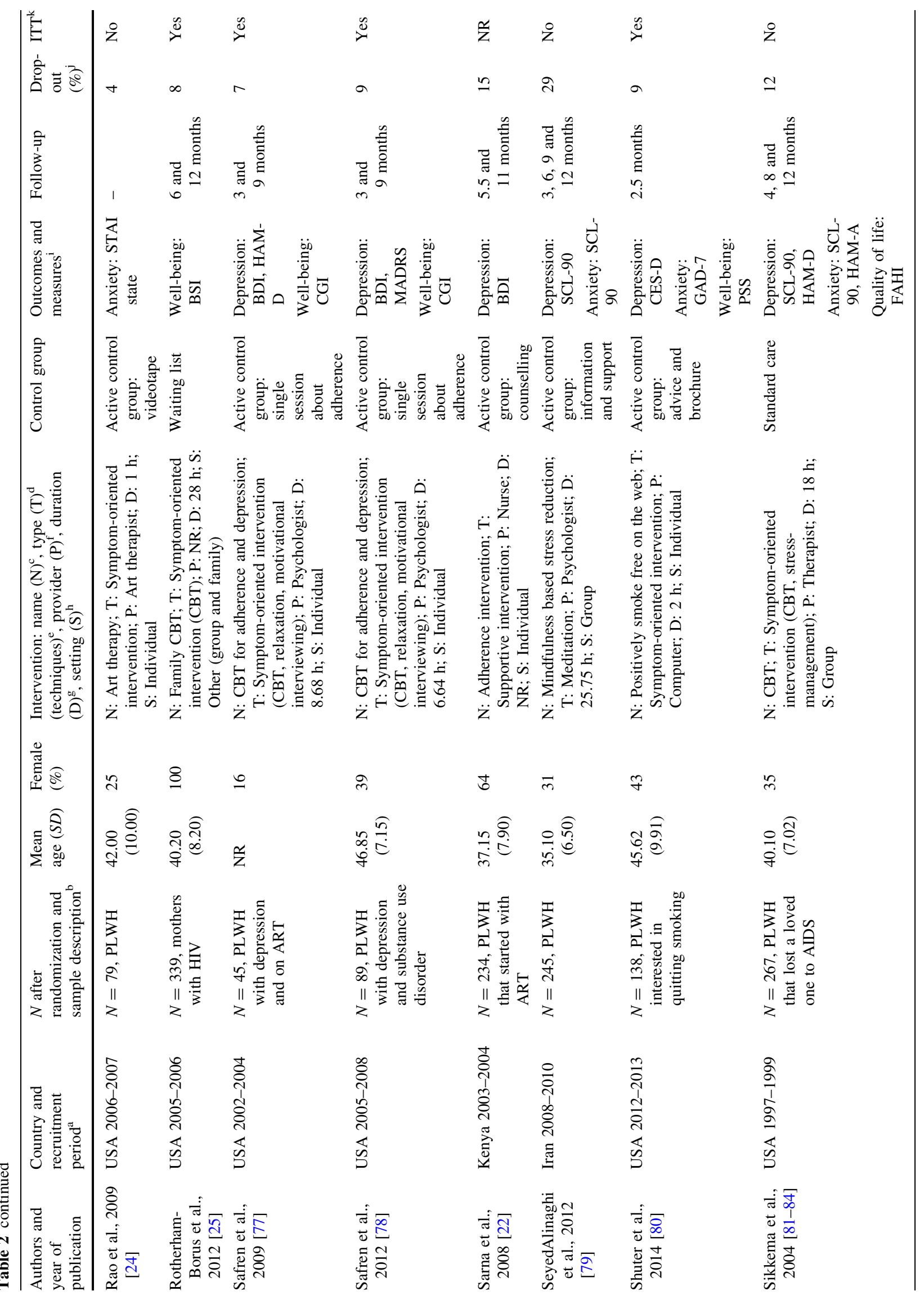




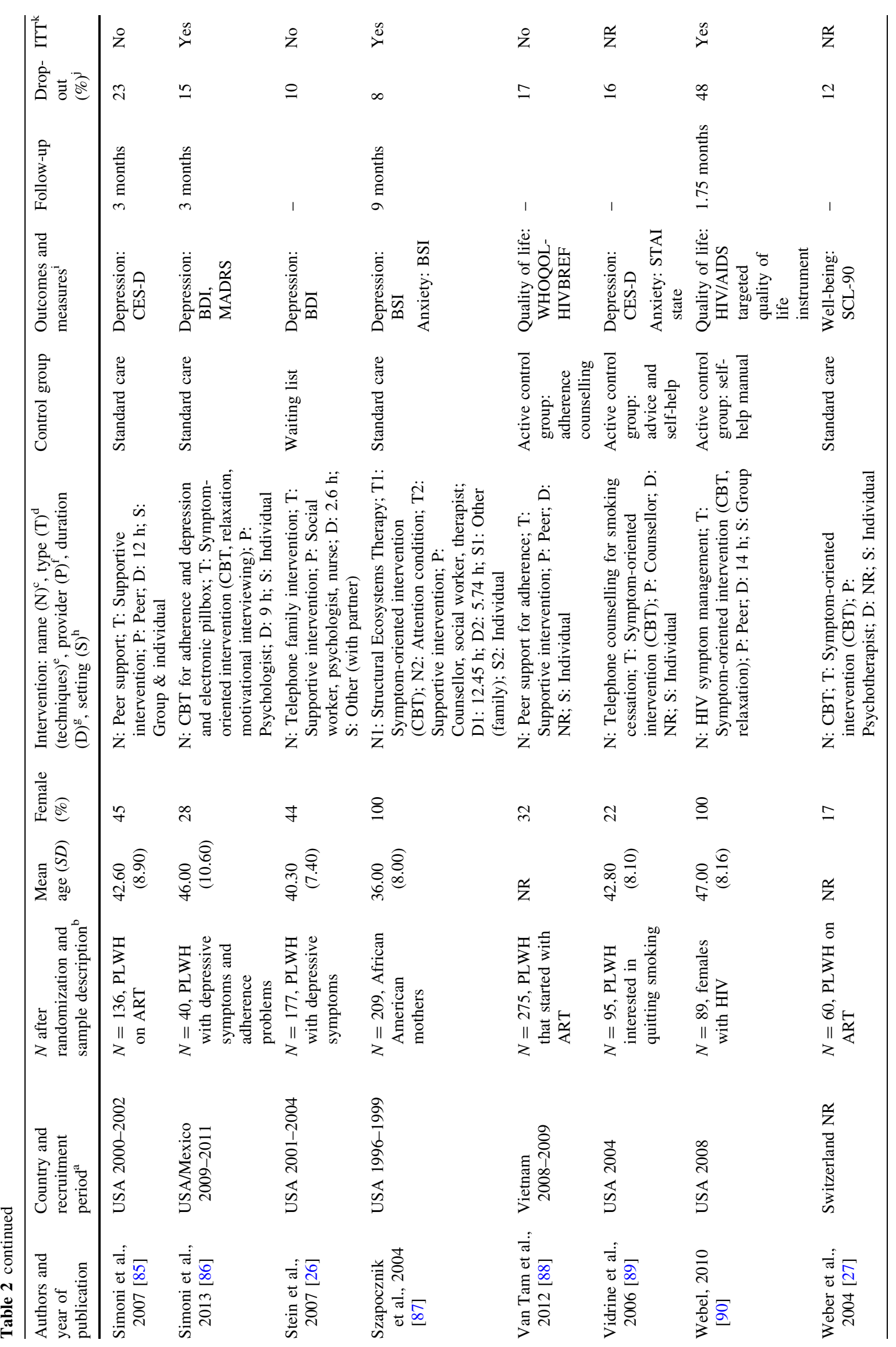




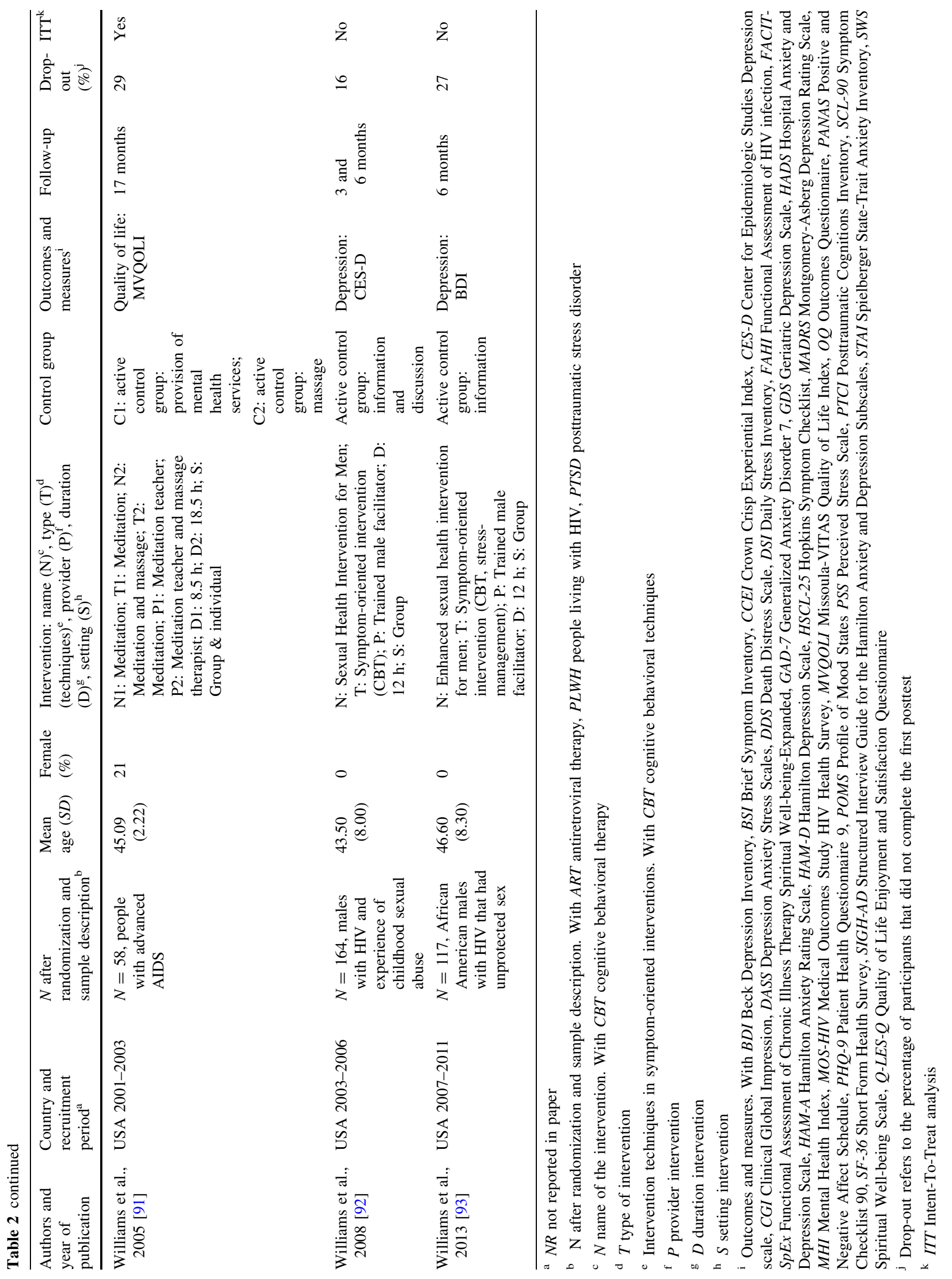


to $3 \mathrm{~h}, m=63$ ). Providers of the interventions were psychologists/psychotherapists (18/65), counsellors (e.g., nurses, 29/65), peers (4/65), none (e.g., computer interventions, 9/65), and other (e.g., investigators, 5/65). The format of the intervention was either individual (31/ 72 ), group (30/72), a combination of individual and group (6/72), or other (e.g., family interventions, 5/72).

\section{Quality of the Included Studies}

The quality ratings of the studies are presented in Table 3 . The first quality criterion, regarding the sequence generation for allocation to conditions, was reported in more than half of the studies (32/62), in the other studies it was unclear. The criterion about the concealment of allocation to conditions was often not reported, only 13 studies mentioned it, in the other studies it was unclear. Incomplete outcome data (the third criterion) was adequately addressed in half of the studies (31/62, e.g., with an ITT analysis), in 20 studies it was not adequately addressed and in 11 studies this was not clear. For most studies (58/62) there was no study protocol available, so the criterion of selective outcome reporting was unclear, only four studies had a rating of low risk of bias on this criterion.

Eight studies investigated an intervention without a provider (e.g., computer or self-help interventions). These studies were not coded on the quality of the administered intervention. In 36 of the 54 studies a treatment manual was available and followed and in 18 studies this was not described. Trained providers were used in 36 of the 54 studies, in 17 studies this was unclear and one study explained that they did not make use of trained providers. Finally, in 31 of the 54 studies treatment integrity was checked and in 23 studies this was not described. A summary of the ratings on all quality criteria is presented in Fig. 2. Regarding the overall quality classification, most studies were classified as low (24/62) or medium (28/62) quality. Only 10 studies were classified as high quality. None of the studies met all quality criteria, but five studies met all except one criterion.

\section{Intervention Effectiveness}

The overall effect of psychosocial interventions on mental health outcomes was Hedges' $\hat{g}=0.19,95 \%$ CI [0.13, 0.25 ], $p<0.001$ (see Fig. 3). Thus, psychosocial interventions may have a positive effect on mental health, compared to a control condition. However, the effect size was small. Standardized residuals were inspected to identify outliers (studies with standardized residuals |3|), but none were found.

Table 4 shows the overall effect of psychosocial interventions on mental health and the effect sizes for each outcome, intervention type and time point separately. It shows that the pooled effect sizes for depression and psychological well-being were larger $(\hat{g}=0.21$ and 0.20$)$ than those for anxiety and quality of life ( $\hat{g}=0.09$ and 0.13$)$. Furthermore, heterogeneity was moderate and significant for the outcomes depression and psychological well-being and smaller and not significant for anxiety and quality of life. Therefore, we decided to conduct the moderator analysis only on the outcomes depression and psychological well-being. Results are presented for the moderator analyses on depression and differences with the moderator analyses of psychological well-being will be discussed.

The results regarding intervention type (categories: symptom-oriented intervention, supportive intervention, and meditation intervention; Table 4) show that each intervention type had a pooled effect size of approximately $\hat{g}=0.20$. The analysis on time points shows that the first and third time point had pooled effect sizes of $\hat{g}=0.18$, while the second $(\hat{g}=0.13)$ and last $(\hat{g}=0.08)$ time point had smaller pooled effect sizes. The pooled effect size of the last time point was not significant and it should be noted that it was based on only nine studies. In sum, the overall effect of psychosocial interventions on mental health outcomes was small $(\hat{g}=0.19)$.

\section{Intervention Effectiveness: Analysis on Last Time Point}

The analysis on outcome type and intervention type described above was also conducted with the last available time point for each study, instead of the first time point. The analysis on the last time point was conducted, as we were also interested in the results on the long term, next to the results on the short term. Differences between those analyses were examined and will be depicted here. The overall effect of psychosocial interventions on mental health was comparable, $\hat{g}=0.18,95 \%$ CI $[0.12,0.25]$, $p<0.001 ; Q=110.25, p<0.001, I^{2}=45 \%$. The pooled effect size on the outcome of anxiety was somewhat larger in this analysis, $\hat{g}=0.14,95 \%$ CI $[0.02,0.25], p<0.05$ and heterogeneity was significant, $Q=39.44, p<0.01$, $I^{2}=47 \%$. The pooled effect size on the outcome psychological well-being was comparable, but heterogeneity was smaller and not significant in this analysis, $Q=34.06$, $p=0.08, I^{2}=30 \%$. Furthermore, the effects of supportive interventions $(\hat{g}=0.18,95 \%$ CI $[0.04,0.33], p<0.05)$ and meditation ( $\hat{g}=0.16,95 \%$ CI $[0.02,0.31], p<0.05$ ) were somewhat smaller in this analysis and the effect of symptom-oriented interventions was larger $(\hat{g}=0.21,95 \%$ CI $[0.14,0.28], p<0.001)$. Summarizing, the analysis on the first time point and the analysis on the last time point were comparable and only small differences were found. 
Table 3 Quality of the included studies

\begin{tabular}{|c|c|c|c|c|c|c|c|c|}
\hline Study & $\begin{array}{l}\text { Sequence } \\
\text { generation }\end{array}$ & $\begin{array}{l}\text { Allocation } \\
\text { concealment }\end{array}$ & $\begin{array}{l}\text { Incomplete } \\
\text { outcome } \\
\text { data }\end{array}$ & $\begin{array}{l}\text { Selective } \\
\text { outcome } \\
\text { reporting }\end{array}$ & $\begin{array}{l}\text { Availability of } \\
\text { treatment } \\
\text { manual }\end{array}$ & $\begin{array}{l}\text { Use of training } \\
\text { for therapy } \\
\text { providers }\end{array}$ & $\begin{array}{l}\text { Treatment } \\
\text { integrity was } \\
\text { checked }\end{array}$ & $\begin{array}{l}\text { Quality } \\
\text { classification }^{\mathrm{a}}\end{array}$ \\
\hline $\begin{array}{l}\text { Balfour et al. } \\
\text { [35] }\end{array}$ & + & + & + & $?$ & + & + & $?$ & High \\
\hline $\begin{array}{l}\text { Berger et al. } \\
{[36]}\end{array}$ & + & + & + & + & + & + & $?$ & High \\
\hline $\begin{array}{l}\text { Boivin et al. } \\
\text { [37] }\end{array}$ & $?$ & $?$ & $?$ & $?$ & $?$ & + & + & Low \\
\hline $\begin{array}{l}\text { Boivin et al. } \\
\text { [38] }\end{array}$ & $?$ & $?$ & + & $?$ & $?$ & + & + & Medium \\
\hline $\begin{array}{l}\text { Bormann et al. } \\
\text { [39] }\end{array}$ & + & $?$ & + & $?$ & + & $?$ & + & Medium \\
\hline $\begin{array}{l}\text { Brazier et al. } \\
\text { [23] }\end{array}$ & + & $?$ & - & $?$ & $?$ & $?$ & $?$ & Low \\
\hline $\begin{array}{l}\text { Brown et al. } \\
{[40]}\end{array}$ & + & $?$ & + & $?$ & NA & NA & NA & Medium \\
\hline $\begin{array}{l}\text { Carrico et al. } \\
\text { [41] }\end{array}$ & + & $?$ & + & $?$ & + & $?$ & + & Medium \\
\hline $\begin{array}{l}\text { Carrico et al. } \\
\text { [42] }\end{array}$ & + & $?$ & + & $?$ & + & $?$ & + & Medium \\
\hline Chan et al. [43] & $?$ & $?$ & - & $?$ & + & $?$ & $?$ & Low \\
\hline $\begin{array}{l}\text { Chang et al. } \\
\text { [44] }\end{array}$ & + & + & + & $?$ & NA & NA & NA & High \\
\hline $\begin{array}{l}\text { Chhatre et al. } \\
{[45]}\end{array}$ & $?$ & $?$ & - & $?$ & $?$ & + & $?$ & Low \\
\hline $\begin{array}{l}\text { Côté and } \\
\text { Pepler [46] }\end{array}$ & $?$ & $?$ & - & $?$ & + & $?$ & + & Low \\
\hline $\begin{array}{l}\text { Duncan et al. } \\
\text { [47] }\end{array}$ & + & $?$ & $?$ & $?$ & $?$ & + & $?$ & Low \\
\hline Eller et al. [48] & $?$ & $?$ & $?$ & $?$ & NA & NA & NA & Low \\
\hline Erlen et al. [49] & $?$ & $?$ & $?$ & $?$ & $?$ & + & + & Low \\
\hline Fife et al. [50] & $?$ & $?$ & - & $?$ & + & $?$ & + & Low \\
\hline $\begin{array}{l}\text { Gayner et al. } \\
\text { [51] }\end{array}$ & + & + & + & $?$ & + & $?$ & $?$ & Medium \\
\hline $\begin{array}{l}\text { Heckman and } \\
\text { Carlson [52] }\end{array}$ & $?$ & $?$ & + & $?$ & + & $?$ & + & Medium \\
\hline $\begin{array}{l}\text { Heckman et al. } \\
\text { [53] }\end{array}$ & + & $?$ & + & $?$ & + & $?$ & + & Medium \\
\hline $\begin{array}{l}\text { Heckman et al. } \\
\text { [54] }\end{array}$ & + & $?$ & + & $?$ & + & - & + & Medium \\
\hline $\begin{array}{l}\text { Hersch et al. } \\
\text { [55] }\end{array}$ & $?$ & $?$ & + & $?$ & NA & NA & NA & Low \\
\hline $\begin{array}{l}\text { Ironson et al. } \\
\text { [56] }\end{array}$ & $?$ & $?$ & + & $?$ & NA & NA & NA & Low \\
\hline $\begin{array}{l}\text { Jensen et al. } \\
\text { [57] }\end{array}$ & $?$ & $?$ & + & $?$ & + & + & + & Medium \\
\hline $\begin{array}{l}\text { Kaaya et al. } \\
{[58]}\end{array}$ & + & + & - & $?$ & $?$ & + & $?$ & Medium \\
\hline $\begin{array}{l}\text { Kalichman } \\
\text { et al. [59] }\end{array}$ & + & + & - & $?$ & + & $?$ & + & Medium \\
\hline $\begin{array}{l}\text { Klein et al. } \\
{[60]}\end{array}$ & + & + & $?$ & $?$ & NA & NA & NA & Medium \\
\hline $\begin{array}{l}\text { Kraaij et al. } \\
{[61]}\end{array}$ & + & $?$ & - & $?$ & NA & NA & NA & Low \\
\hline
\end{tabular}


Table 3 continued

\begin{tabular}{|c|c|c|c|c|c|c|c|c|}
\hline Study & $\begin{array}{l}\text { Sequence } \\
\text { generation }\end{array}$ & $\begin{array}{l}\text { Allocation } \\
\text { concealment }\end{array}$ & $\begin{array}{l}\text { Incomplete } \\
\text { outcome } \\
\text { data }\end{array}$ & $\begin{array}{l}\text { Selective } \\
\text { outcome } \\
\text { reporting }\end{array}$ & $\begin{array}{l}\text { Availability of } \\
\text { treatment } \\
\text { manual }\end{array}$ & $\begin{array}{l}\text { Use of training } \\
\text { for therapy } \\
\text { providers }\end{array}$ & $\begin{array}{l}\text { Treatment } \\
\text { integrity was } \\
\text { checked }\end{array}$ & $\begin{array}{l}\text { Quality } \\
\text { classification }^{\mathrm{a}}\end{array}$ \\
\hline $\begin{array}{l}\text { Lechner et al. } \\
\text { [62] }\end{array}$ & $?$ & $?$ & - & $?$ & + & + & + & Medium \\
\hline Li et al. [63] & $?$ & $?$ & $?$ & $?$ & + & + & $?$ & Low \\
\hline Lovejoy [64] & + & + & + & $?$ & + & + & + & High \\
\hline $\begin{array}{l}\text { McCain et al. } \\
\text { [65] }\end{array}$ & + & + & + & $?$ & + & + & $?$ & High \\
\hline $\begin{array}{l}\text { Miles et al. } \\
\text { [66] }\end{array}$ & + & $?$ & + & $?$ & $?$ & + & + & Medium \\
\hline $\begin{array}{l}\text { Miller et al. } \\
\text { [67] }\end{array}$ & $?$ & $?$ & - & $?$ & $?$ & + & $?$ & Low \\
\hline $\begin{array}{l}\text { Mitrani et al. } \\
\text { [68] }\end{array}$ & $?$ & $?$ & + & $?$ & + & + & + & Medium \\
\hline $\begin{array}{l}\text { Murphy et al. } \\
\text { [69] }\end{array}$ & + & $?$ & - & $?$ & + & $?$ & $?$ & Low \\
\hline $\begin{array}{l}\text { Murphy et al. } \\
{[70]}\end{array}$ & $?$ & $?$ & + & $?$ & + & $?$ & $?$ & Low \\
\hline $\begin{array}{l}\text { O'Leary et al. } \\
\text { [71] }\end{array}$ & + & $?$ & - & $?$ & + & + & $?$ & Medium \\
\hline Olley [72] & $?$ & $?$ & $?$ & $?$ & + & $?$ & $?$ & Low \\
\hline $\begin{array}{l}\text { Pacella et al. } \\
\text { [73] }\end{array}$ & $?$ & $?$ & + & $?$ & + & + & + & Medium \\
\hline $\begin{array}{l}\text { Peltzer et al. } \\
\text { [74] }\end{array}$ & + & $?$ & $?$ & $?$ & + & $?$ & $?$ & Low \\
\hline $\begin{array}{l}\text { Petersen et al. } \\
\text { [75] }\end{array}$ & + & $?$ & - & $?$ & + & + & + & Medium \\
\hline $\begin{array}{l}\text { Ransom et al. } \\
\text { [76] }\end{array}$ & $?$ & $?$ & + & $?$ & + & + & + & Medium \\
\hline Rao et al. [24] & $?$ & $?$ & + & $?$ & $?$ & $?$ & $?$ & Low \\
\hline $\begin{array}{l}\text { Rotherham- } \\
\text { Borus et al. } \\
\text { [25] }\end{array}$ & $?$ & $?$ & + & + & + & + & + & High \\
\hline $\begin{array}{l}\text { Safren et al. } \\
\text { [77] }\end{array}$ & $?$ & $?$ & + & $?$ & + & $?$ & + & Medium \\
\hline $\begin{array}{l}\text { Safren et al. } \\
\text { [78] }\end{array}$ & $?$ & $?$ & + & $?$ & + & + & + & Medium \\
\hline $\begin{array}{l}\text { Sarna et al. } \\
\text { [22] }\end{array}$ & + & + & $?$ & $?$ & $?$ & + & + & Medium \\
\hline $\begin{array}{l}\text { SeyedAlinaghi } \\
\text { et al. [79] }\end{array}$ & + & $?$ & - & + & + & + & $?$ & Medium \\
\hline $\begin{array}{l}\text { Shuter et al. } \\
\text { [80] }\end{array}$ & + & $?$ & + & + & NA & NA & NA & High \\
\hline $\begin{array}{l}\text { Sikkema et al. } \\
\text { [81-84] }\end{array}$ & $?$ & $?$ & - & $?$ & + & + & + & Medium \\
\hline $\begin{array}{l}\text { Simoni et al. } \\
{[85]}\end{array}$ & + & + & - & $?$ & $?$ & + & + & Medium \\
\hline $\begin{array}{l}\text { Simoni et al. } \\
{[86]}\end{array}$ & + & + & + & $?$ & + & + & + & High \\
\hline Stein et al. [26] & $?$ & $?$ & - & $?$ & + & + & + & Medium \\
\hline $\begin{array}{r}\text { Szapocznik } \\
\text { et al. [87] }\end{array}$ & + & $?$ & + & $?$ & + & + & + & High \\
\hline $\begin{array}{l}\text { Van Tam et al. } \\
{[88]}\end{array}$ & $?$ & $?$ & - & $?$ & $?$ & + & + & Low \\
\hline
\end{tabular}


Table 3 continued

\begin{tabular}{|c|c|c|c|c|c|c|c|c|}
\hline Study & $\begin{array}{l}\text { Sequence } \\
\text { generation }\end{array}$ & $\begin{array}{l}\text { Allocation } \\
\text { concealment }\end{array}$ & $\begin{array}{l}\text { Incomplete } \\
\text { outcome } \\
\text { data }\end{array}$ & $\begin{array}{l}\text { Selective } \\
\text { outcome } \\
\text { reporting }\end{array}$ & $\begin{array}{l}\text { Availability of } \\
\text { treatment } \\
\text { manual }\end{array}$ & $\begin{array}{l}\text { Use of training } \\
\text { for therapy } \\
\text { providers }\end{array}$ & $\begin{array}{l}\text { Treatment } \\
\text { integrity was } \\
\text { checked }\end{array}$ & $\begin{array}{l}\text { Quality } \\
\text { classification }^{\mathrm{a}}\end{array}$ \\
\hline $\begin{array}{l}\text { Vidrine et al. } \\
\text { [89] }\end{array}$ & + & $?$ & $?$ & $?$ & $?$ & + & $?$ & Low \\
\hline Webel [90] & $?$ & $?$ & + & $?$ & $?$ & + & $?$ & Low \\
\hline $\begin{array}{l}\text { Weber et al. } \\
\text { [27] }\end{array}$ & + & + & $?$ & $?$ & + & + & + & High \\
\hline $\begin{array}{l}\text { Williams et al. } \\
\text { [91] }\end{array}$ & + & $?$ & + & $?$ & $?$ & + & $?$ & Medium \\
\hline $\begin{array}{l}\text { Williams et al. } \\
\text { [92] }\end{array}$ & $?$ & $?$ & - & $?$ & $?$ & + & $?$ & Low \\
\hline $\begin{array}{l}\text { Williams et al. } \\
\text { [93] }\end{array}$ & $?$ & $?$ & - & $?$ & $?$ & + & $?$ & Low \\
\hline
\end{tabular}

+ low risk of bias, - high risk of bias; ? unclear risk of bias; NA = not applicable (e.g., when the intervention is a self-help program)

a quality classification, this was calculated by adding up the low risk of bias ratings (see "Method" section)

Fig. 2 Risk of bias graph



\section{Publication Bias}

When the funnel plot was inspected (see Fig. 4), it was clear that studies were missing on the left side of the plot. This may be an indication of publication bias. Egger's test of the intercept was significant, intercept $0.82,95 \%$ CI $[0.09,1.54], t(60)=2.24, p<0.05$. This also indicates that there may be publication bias. Lastly, Duval and Tweedie's trim and fill analysis demonstrated that 14 studies were missing on the left side of the plot (see black dots in Fig. 4). After imputation of these 14 studies, the adjusted effect size was $\hat{g}=0.11,95 \%$ CI [0.04, 0.17]. This effect size is substantially smaller than the unadjusted effect size of $\hat{g}=0.19$. In sum, there seems to be evidence for publication bias in this meta-analysis, as studies with smaller effect sizes are missing.

\section{Moderator Analysis on the Outcome Depression}

Table 5 shows the effects of the subgroup analysis on the outcome depression. It shows that the type of control group was a significant moderator. Contrary to expectations, studies that used a waiting list control group had smaller effect sizes in general, than studies that used an active or standard care control condition. However, there were only five studies in this analysis that used a waiting list control group. Also, the percentage of people with AIDS in a study was a significant moderator, i.e., when less than $40 \%$ of the 
$\underline{\text { Study }}$

Balfour et al., 2006 Berger et al., 2008 Boivin et al., 2013a

Boivin et al., 2013b

Bormann et al., 2006

Brazier et al., 2006

Brown et al., 2011

Carrico et al., 2006

Carrico et al., 2009

Chan et al., 2005

Chang et al., 2007

Chhatre et al., 2013

Côté \& Pepler, 2002

Duncan et al., 2012

Eller et al., 2013

Erlen et al., 2001

Fife et al., 2008

Gayner et al., 2012

Heckman \& Carlson, 2007

Heckman et al., 2011

Heckman et al., 2013

Hersch et al., 2013

Ironson et al., 2013

Jensen et al., 2013

Kaaya et al., 2013

Kalichman, 2005

Klein et al., 2013

Kraaij et al., 2010

Lechner et al., 2003

Li et al., 2010

Lovejoy, 2012

McCain et al., 2008

Miles et al., 2003

Miller et al., 2005

Mitrani et al., 2012

Murphy et al., 2002

Murphy et al., 2011

O'Leary et al., 2005

Olley, 2006

Pacella et al., 2012

Peltzer et al., 2012

Petersen et al., 2014

Ransom et al., 2008

Rao et al., 2009

Rotherham-Borus et al., 2012

Safren et al., 2009

Safren et al., 2012

Sarna et al., 2008

SeyedAlinaghi et al., 2012

Shuter et al., 2014

Sikkema et al., 2004

Simoni et al., 2007

Simoni et al., 2013

Stein et al., 2007

Szapocznik et al., 2004

Van Tam et al., 2012

Vidrine et al., 2006

Webel, 2010

Weber et al., 2004

Williams et al., 2005

Williams et al., 2008

Williams et al., 2013

Overall effect

\begin{tabular}{|c|c|c|}
\hline $\begin{array}{c}\text { Hedges's } \\
\text { g }\end{array}$ & $\begin{array}{c}\text { Lower } \\
\text { limit }\end{array}$ & $\begin{array}{c}\text { Upper } \\
\text { limit }\end{array}$ \\
\hline 1,059 & 0,270 & 1,847 \\
\hline 0,634 & 0,178 & 1,089 \\
\hline$-0,077$ & $-0,581$ & 0,427 \\
\hline$-0,070$ & $-0,443$ & 0,303 \\
\hline 0,077 & $-0,382$ & 0,535 \\
\hline 0,000 & $-0,569$ & 0,569 \\
\hline$-0,190$ & $-0,695$ & 0,315 \\
\hline 0,465 & 0,047 & 0,882 \\
\hline$-0,018$ & $-0,145$ & 0,110 \\
\hline 0,812 & $-0,249$ & 1,873 \\
\hline 0,227 & $-0,210$ & 0,665 \\
\hline 0,430 & $-0,426$ & 1,285 \\
\hline 0,290 & $-0,231$ & 0,811 \\
\hline 0,130 & $-0,354$ & 0,615 \\
\hline 0,710 & 0,412 & 1,008 \\
\hline$-0,320$ & $-1,167$ & 0,526 \\
\hline 0,177 & $-0,448$ & 0,802 \\
\hline 0,327 & $-0,057$ & 0,711 \\
\hline 0,115 & $-0,161$ & 0,391 \\
\hline 0,486 & 0,197 & 0,774 \\
\hline 0,464 & 0,208 & 0,719 \\
\hline 0,074 & $-0,253$ & 0,401 \\
\hline$-0,019$ & $-0,288$ & 0,250 \\
\hline 0,234 & $-0,250$ & 0,717 \\
\hline 0,324 & $-0,015$ & 0,662 \\
\hline 0,241 & $-0,196$ & 0,679 \\
\hline 0,423 & 0,119 & 0,728 \\
\hline 0,382 & $-0,330$ & 1,095 \\
\hline 0,271 & 0,054 & 0,488 \\
\hline 0,022 & $-0,154$ & 0,198 \\
\hline$-0,232$ & $-0,744$ & 0,280 \\
\hline 0,198 & $-0,156$ & 0,552 \\
\hline 0,281 & $-0,172$ & 0,735 \\
\hline 0,454 & $-0,740$ & 1,648 \\
\hline$-0,106$ & $-0,522$ & 0,310 \\
\hline 0,321 & $-0,355$ & 0,997 \\
\hline$-0,061$ & $-0,528$ & 0,406 \\
\hline 0,166 & 0,016 & 0,315 \\
\hline 0,428 & $-0,072$ & 0,929 \\
\hline 0,527 & 0,025 & 1,029 \\
\hline 0,050 & $-0,267$ & 0,366 \\
\hline 0,578 & $-0,096$ & 1,251 \\
\hline 0,322 & $-0,118$ & 0,762 \\
\hline 0,000 & $-0,437$ & 0,437 \\
\hline 0,000 & $-0,212$ & 0,212 \\
\hline 0,859 & 0,257 & 1,461 \\
\hline 0,636 & 0,213 & 1,059 \\
\hline 0,215 & $-0,063$ & 0,494 \\
\hline 0,196 & $-0,102$ & 0,493 \\
\hline$-0,054$ & $-0,401$ & 0,293 \\
\hline 0,189 & $-0,150$ & 0,527 \\
\hline$-0,076$ & $-0,456$ & 0,304 \\
\hline 0,327 & $-0,285$ & 0,939 \\
\hline$-0,106$ & $-0,415$ & 0,202 \\
\hline 0,210 & $-0,132$ & 0,552 \\
\hline$-0,002$ & $-0,370$ & 0,366 \\
\hline 0,429 & $-0,019$ & 0,876 \\
\hline$-0,446$ & $-1,039$ & 0,148 \\
\hline 0,000 & $-0,533$ & 0,533 \\
\hline 0,451 & $-0,280$ & 1,182 \\
\hline$-0,030$ & $-0,365$ & 0,304 \\
\hline 0,277 & $-0,139$ & 0,694 \\
\hline 0,187 & 0,126 & 0,249 \\
\hline
\end{tabular}

Hedges's g and $95 \% \mathrm{Cl}$

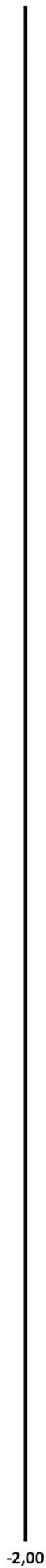

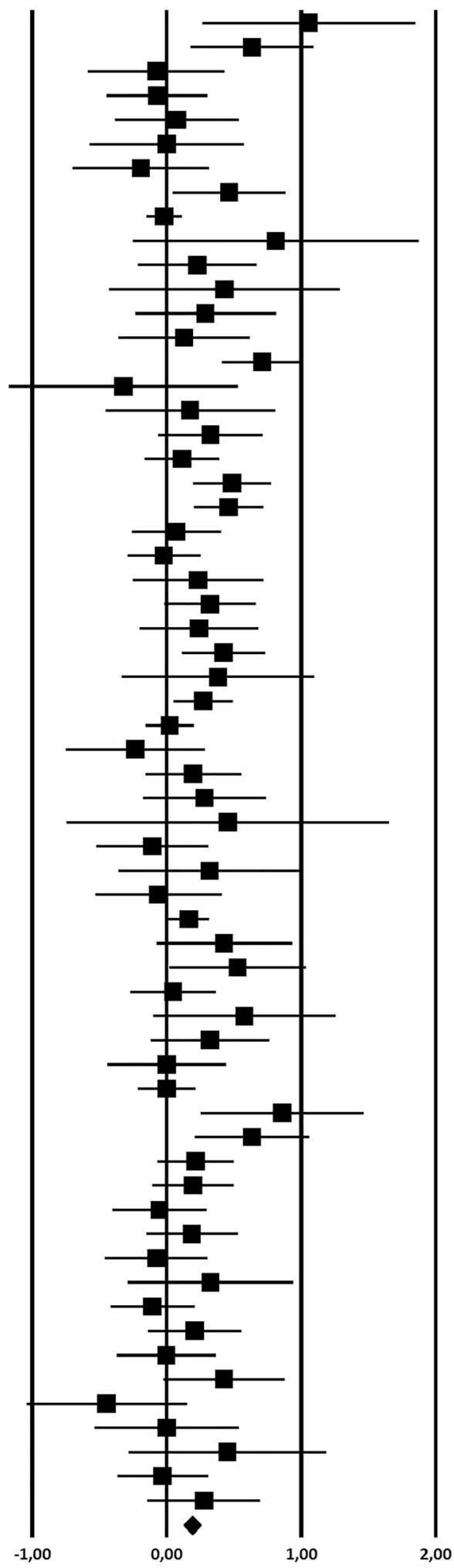

Favours control

Favours intervention

Fig. 3 Forest plot showing the effect of psychosocial interventions on mental health outcomes 
Table 4 Overall analysis and analysis separately for each outcome, intervention type and time point

\begin{tabular}{lllllll}
\hline Analysis & Subgroup & $k^{\mathrm{a}}$ & Hedges'$^{\prime} \hat{g}$ & $95 \% \mathrm{CI}$ & $Q^{\mathrm{b}}$ & $I^{2}(\%)^{\mathrm{c}}$ \\
\hline Overall effect & & 62 & $0.19^{\mathrm{d}}$ & $0.13,0.25$ & $99.35^{\mathrm{d}}$ & 39 \\
Outcome & Depression & 47 & $0.21^{\mathrm{d}}$ & $0.13,0.29$ & $87.32^{\mathrm{d}}$ & 47 \\
& Anxiety & 22 & 0.09 & $-0.01,0.19$ & 31.29 & 33 \\
& Quality of life & 19 & $0.13^{\mathrm{d}}$ & $0.04,0.21$ & 21.07 & 15 \\
& Psychological well-being & 25 & $0.20^{\mathrm{d}}$ & $0.09,0.31$ & $44.63^{\mathrm{d}}$ & 46 \\
Intervention type & Symptom-oriented intervention & 39 & $0.19^{\mathrm{d}}$ & $0.11,0.28$ & $69.71^{\mathrm{d}}$ & 46 \\
& Supportive intervention & 20 & $0.21^{\mathrm{d}}$ & $0.09,0.33$ & $43.58^{\mathrm{d}}$ & 56 \\
& Meditation intervention & 9 & $0.20^{\mathrm{d}}$ & $0.06,0.35$ & 2.00 & 0 \\
Time point & Posttest 0-3 months & 59 & $0.18^{\mathrm{d}}$ & $0.12,0.25$ & $85.79^{\mathrm{e}}$ & 32 \\
& Posttest 3-6 months & 19 & $0.13^{\mathrm{d}}$ & $0.05,0.22$ & 28.31 & 36 \\
& Posttest 6-9 months & 12 & $0.18^{\mathrm{d}}$ & $0.05,0.31$ & $22.97^{\mathrm{e}}$ & 52 \\
& Posttest $>9$ months & 9 & 0.08 & $-0.05,0.21$ & 13.79 & 42 \\
\hline
\end{tabular}

${ }^{\mathrm{a}} k=$ number of studies

${ }^{\text {b }} Q=$ heterogeneity test

c $I^{2}=\%$ of heterogeneity

d $p<0.01$

e $p<0.05$
Fig. 4 Funnel plot of standard error by Hedges's $g$ with imputed studies from Duval and Tweedie's trim and fill analysis

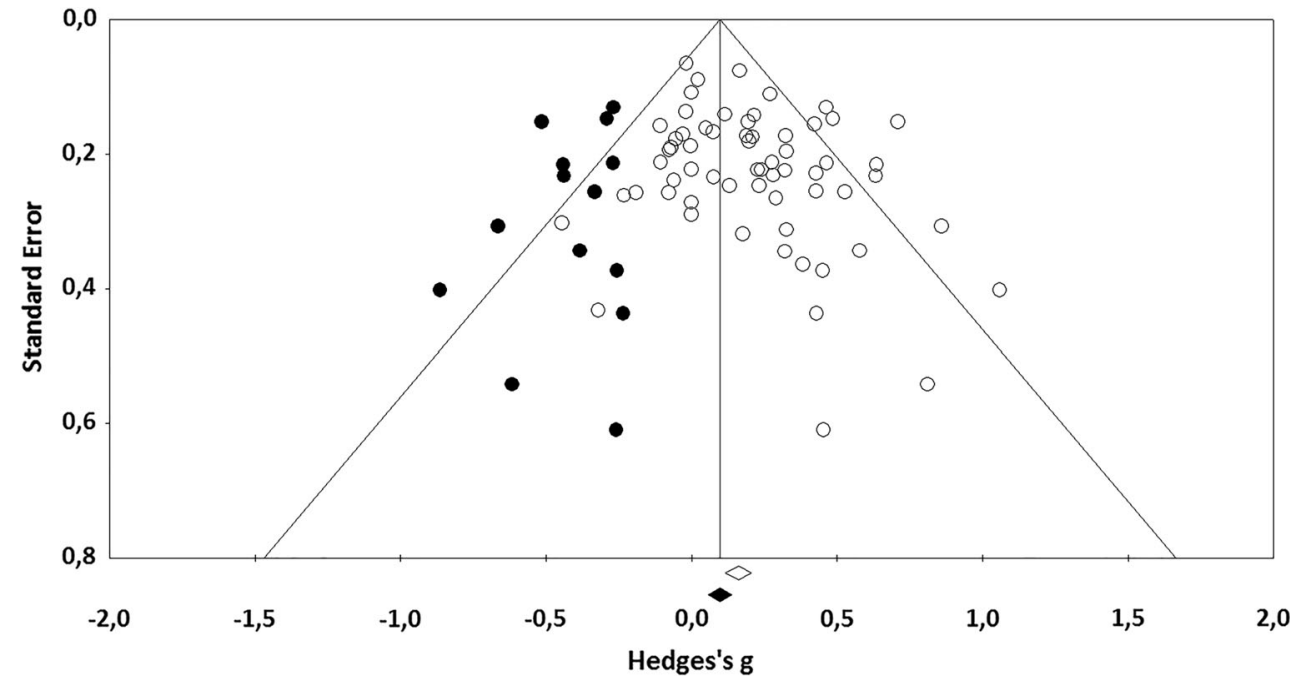

participants in the studies had AIDS the effect sizes were on average larger than when $40 \%$ or more of the participants in the studies had AIDS. Furthermore, the moderator a priori screening on depression was significant, which means that studies that only included participants with depressive symptoms had larger effect sizes in general ( $\hat{g}=0.46,95 \%$ CI $[0.25,0.68])$, than studies that did not had the presence of depressive symptoms as an inclusion criterion $(\hat{g}=0.12,95 \%$ CI $[0.05,0.18])$. Last, the moderator provider of the intervention was significant. Studies that had a psychologist or a psychotherapist as a provider of the intervention had the largest pooled effect sizes.
Studies that used other providers (e.g., a counsellor or peer or a computer) had smaller pooled effect sizes. Concluding, the moderators that were found to be significant were: type of control group, percentage of people with AIDS, a priori screening on depression, and provider of the intervention.

\section{Moderator Analysis: Effect of Intervention Characteristics on the Outcome Depression}

Table 6 shows the effects of intervention characteristics on the outcome of depression. In this analysis, all interventions were investigated separately, so some studies are 
Table 5 Moderators of intervention effect on depression $(k=47)$

\begin{tabular}{|c|c|c|c|c|c|}
\hline Moderator & Subgroup & $k^{\mathrm{a}}$ & Hedges' $\hat{g}$ & $95 \% \mathrm{CI}$ & $Q$ for difference $\mathrm{e}^{\mathrm{b}}$ \\
\hline \multirow[t]{3}{*}{ Control group } & Active control group & 24 & $0.22^{\mathrm{c}}$ & $0.11,0.33$ & $6.74^{\mathrm{d}}$ \\
\hline & Standard care & 18 & $0.25^{\mathrm{c}}$ & $0.14,0.36$ & \\
\hline & Waiting list & 5 & -0.04 & $-0.24,0.16$ & \\
\hline \multirow[t]{3}{*}{ Location } & Africa/Asia & 8 & $0.19^{c}$ & $0.05,0.33$ & 0.01 \\
\hline & North America/Europe & 38 & $0.19^{\mathrm{c}}$ & $0.10,0.27$ & \\
\hline & Other $^{\mathrm{e}}$ & 1 & & & \\
\hline \multirow[t]{4}{*}{ First year recruitment } & 1996-2001 & 16 & $0.13^{\mathrm{d}}$ & $0.02,0.24$ & 4.37 \\
\hline & $2002-2006$ & 15 & $0.32^{\mathrm{c}}$ & $0.16,0.47$ & \\
\hline & 2007-2012 & 13 & 0.13 & $-0.01,0.28$ & \\
\hline & Missing & 3 & & & \\
\hline \multirow[t]{4}{*}{$\%$ Attrition } & $0-10 \%$ & 14 & $0.18^{\mathrm{d}}$ & $0.01,0.36$ & 1.14 \\
\hline & $10-20 \%$ & 17 & $0.20^{\mathrm{c}}$ & $0.08,0.32$ & \\
\hline & $>20 \%$ & 14 & $0.28^{\mathrm{c}}$ & $0.15,0.41$ & \\
\hline & Missing & 2 & 0.03 & & \\
\hline \multirow[t]{4}{*}{$\%$ Females } & $0-20 \%$ & 14 & $0.27^{\mathrm{c}}$ & $0.15,0.40$ & 4.54 \\
\hline & $20-80 \%$ & 22 & $0.21^{\mathrm{c}}$ & $0.09,0.33$ & \\
\hline & $80-100 \%$ & 10 & 0.08 & $-0.06,0.21$ & \\
\hline & Missing & 1 & & & \\
\hline \multirow[t]{3}{*}{ Mean age } & $<42.40$ years & 19 & $0.14^{\mathrm{c}}$ & $0.04,0.24$ & 0.74 \\
\hline & $\geq 42.40$ years & 22 & $0.21^{\mathrm{c}}$ & $0.09,0.32$ & \\
\hline & Missing & 6 & & & \\
\hline \multirow[t]{3}{*}{$\% \mathrm{MSM}^{\mathrm{f}}$} & $0 \%$ & 10 & 0.10 & $-0.05,0.24$ & 0.50 \\
\hline & $>0 \%$ & 10 & $0.17^{\mathrm{d}}$ & $0.02,0.31$ & \\
\hline & Missing & 27 & & & \\
\hline \multirow[t]{3}{*}{$\%$ Participants with AIDS } & $<40 \%$ & 5 & $0.54^{\mathrm{c}}$ & $0.38,0.71$ & $7.24^{\mathrm{c}}$ \\
\hline & $\geq 40 \%$ & 5 & 0.19 & $-0.01,0.38$ & \\
\hline & Missing & 37 & & & \\
\hline \multirow[t]{3}{*}{ Mean duration HIV } & $<10.02$ years & 10 & 0.16 & $-0.05,0.38$ & 0.21 \\
\hline & $\geq 10.02$ years & 13 & $0.22^{\mathrm{c}}$ & $0.07,0.38$ & \\
\hline & Missing & 24 & & & \\
\hline \multirow[t]{3}{*}{$\%$ on $\mathrm{ART}^{\mathrm{g}}$} & $<87 \%$ & 10 & $0.34^{\mathrm{c}}$ & $0.15,0.52$ & 0.00 \\
\hline & $\geq 87 \%$ & 12 & $0.34^{\mathrm{c}}$ & $0.18,0.49$ & \\
\hline & Missing & 25 & & & \\
\hline \multirow[t]{2}{*}{ Screening on depression } & No & 37 & $0.12^{\mathrm{c}}$ & $0.05,0.18$ & $9.13^{\mathrm{c}}$ \\
\hline & Yes & 10 & $0.46^{\mathrm{c}}$ & $0.25,0.68$ & \\
\hline \multirow[t]{2}{*}{ Mental health primary outcome } & No & 6 & 0.17 & $-0.02,0.36$ & 0.19 \\
\hline & Yes & 41 & $0.22^{\mathrm{c}}$ & $0.13,0.30$ & \\
\hline \multirow[t]{6}{*}{ Provider intervention } & Psychologist & 13 & $0.42^{\mathrm{c}}$ & $0.28,0.56$ & $12.92^{\mathrm{d}}$ \\
\hline & Counsellor & 21 & $0.15^{\mathrm{c}}$ & $0.05,0.25$ & \\
\hline & Peer & 2 & 0.10 & $-0.04,0.25$ & \\
\hline & None & 5 & 0.18 & $-0.19,0.54$ & \\
\hline & Other (practitioner) & 1 & 0.06 & $-0.22,0.33$ & \\
\hline & Missing & 5 & & & \\
\hline \multirow[t]{4}{*}{ Format intervention } & Group & 19 & $0.23^{\mathrm{c}}$ & $0.14,0.33$ & 4.58 \\
\hline & Individual & 20 & $0.25^{\mathrm{c}}$ & $0.10,0.40$ & \\
\hline & Combination & 4 & 0.09 & $-0.16,0.33$ & \\
\hline & Other & 4 & -0.01 & $-0.26,0.23$ & \\
\hline Analysis & ITT $^{\mathrm{h}}$ & 24 & $0.22^{\mathrm{c}}$ & $0.11,0.34$ & 1.32 \\
\hline
\end{tabular}


Table 5 continued

\begin{tabular}{llllll}
\hline Moderator & Subgroup & $k^{\mathrm{a}}$ & Hedges' $^{\prime}$ & $95 \%$ CI & $Q$ for difference $^{\mathrm{b}}$ \\
\hline & No ITT & 15 & $0.13^{\mathrm{d}}$ & $0.02,0.24$ \\
Missing & 8 & & & $0.08,0.38$ \\
Study quality & Low & 17 & $0.23^{\mathrm{c}}$ & $0.09,0.29$ \\
& Medium & 24 & $0.19^{\mathrm{c}}$ & $0.001,0.57$ \\
\hline
\end{tabular}

${ }^{\mathrm{a}} k=$ number of studies

${ }^{\text {b }} Q=Q$ for difference between subgroups

c $p<0.01$

${ }^{\mathrm{d}} p<0.05$

e One study recruited participants in South Africa, Puerto Rico and the USA. This study could not be classified into one of the categories, so it was removed from the moderator analysis on location

${ }^{\mathrm{f}} M S M$ men that have sex with men

g $A R T$ antiretroviral therapy

${ }^{\mathrm{h}}$ ITT intent to treat analysis

represented twice. When mental health (i.e., depression, anxiety, quality of life, or psychological well-being) was a primary focus of the intervention in a study, the effect sizes were in general larger than when mental health was not a primary focus of an intervention. Furthermore, treatment duration was a significant moderator. Studies with treatment durations of $12-18 \mathrm{~h}$ had the largest effect sizes. Studies with shorter or longer treatment durations had smaller effect sizes in general. In sum, the following moderators were significant in this analysis: mental health primary focus of the intervention and treatment duration.

\section{Moderator Analysis on the Outcome Psychological Well-Being}

The moderator analysis was also conducted on the outcome psychological well-being, next to the moderator analysis on the outcome depression. It was decided to do the moderator analysis on these two outcomes, because the largest pooled effect sizes were found for depression and psychological well-being and heterogeneity was highest and significant for these outcomes (see Table 4). Differences between the moderator analysis on the outcome depression and psychological well-being will be discussed here. The moderators percentage of people with AIDS, provider of the intervention, whether mental health was a primary focus of the intervention and treatment duration were not significant in the analysis on well-being, all $p$ 's $>0.06$. The type of control group remained a significant moderator. The moderator screening on the presence of depressive symptoms was not included in this analysis, since the outcome was psychological well-being and therefore most studies did not screen on depressive symptoms in these studies. Furthermore, the percentage of people on ART ( $Q=4.10$, $p<0.05)$ and study quality $(Q=8.71, p<0.05)$ were significant moderators in this analysis. Regarding the percentage of people on ART, the largest effect sizes were in general for studies with $87 \%$ or more of the participants on ART $(\hat{g}=0.39,95 \%$ CI $[0.14,0.64], p<0.01, k=8)$, and studies with less than $87 \%$ of participants on ART had smaller effect sizes $(\hat{g}=0.07,95 \%$ CI $[-0.11,0.25]$, $p=0.45, k=3)$. Though, the last category contained only three studies. Regarding study quality, studies with a medium quality had larger effect sizes on average $(\hat{g}=0.30,95 \%$ CI $[0.14,0.46], p<0.001, k=14)$, than studies with a low $(\hat{g}=0.15,95 \%$ CI $[-0.05,0.35]$, $p=0.15, k=7)$ or high quality $(\hat{g}=-0.04,95 \% \mathrm{CI}$ $[-0.21,0.12], p=0.61, k=4)$. However, there were only four studies in the category high quality in this analysis. Summarizing, in the moderator analysis on the outcome psychological well-being the significant moderators were: type of control group, percentage of people on ART, and study quality.

\section{Discussion}

The first aim of this systematic review and meta-analysis was to investigate the effectiveness for PLWH of various psychosocial interventions aimed at decreasing depression and anxiety, and improving quality of life and psychological well-being, and to investigate which interventions were the most effective on these outcomes. Sixty-two studies were included in the analysis, and it was found that psychosocial interventions had a positive effect on the mental health outcomes described, although the effect size was small. In addition, there was evidence of publication bias, so the corrected effect size was smaller. Most studies in the 
Table 6 Effect of intervention characteristics on depression

\begin{tabular}{|c|c|c|c|c|c|}
\hline Moderator & Subgroup & $m^{\mathrm{a}}$ & Hedges' $\hat{g}$ & $95 \% \mathrm{CI}$ & $Q$ for difference ${ }^{\mathrm{b}}$ \\
\hline \multirow[t]{2}{*}{ Relaxation technique $^{\mathrm{c}}$} & No & 22 & $0.18^{\mathrm{d}}$ & $0.06,0.29$ & \multirow[t]{2}{*}{2.75} \\
\hline & Yes & 9 & $0.38^{\mathrm{d}}$ & $0.17,0.59$ & \\
\hline \multirow[t]{2}{*}{ Cognitive behavioral technique ${ }^{c}$} & No & 9 & 0.15 & $-0.07,0.38$ & \multirow[t]{2}{*}{0.53} \\
\hline & Yes & 22 & $0.25^{\mathrm{d}}$ & $0.13,0.37$ & \\
\hline \multirow[t]{2}{*}{ Motivational interviewing technique $^{c}$} & No & 25 & $0.23^{\mathrm{d}}$ & $0.12,0.34$ & \multirow[t]{2}{*}{0.07} \\
\hline & Yes & 6 & 0.18 & $-0.16,0.52$ & \\
\hline \multirow[t]{2}{*}{ Stress-management technique ${ }^{c}$} & No & 20 & $0.19^{\mathrm{d}}$ & $0.06,0.31$ & \multirow[t]{2}{*}{1.04} \\
\hline & Yes & 11 & $0.29^{\mathrm{d}}$ & $0.13,0.45$ & \\
\hline \multirow[t]{2}{*}{ Mental health primary focus intervention } & No & 22 & $0.14^{\mathrm{d}}$ & $0.06,0.22$ & \multirow[t]{2}{*}{$4.02^{\mathrm{e}}$} \\
\hline & Yes & 31 & $0.29^{\mathrm{d}}$ & $0.02,0.41$ & \\
\hline \multirow[t]{2}{*}{ Theory-driven intervention } & No & 19 & $0.22^{\mathrm{d}}$ & $0.09,0.36$ & \multirow[t]{2}{*}{0.01} \\
\hline & Yes & 34 & $0.21^{\mathrm{d}}$ & $0.12,0.31$ & \\
\hline \multirow[t]{5}{*}{ Treatment duration } & $1-5 \mathrm{~h}$ & 13 & 0.06 & $-0.09,0.20$ & \multirow[t]{5}{*}{$10.76^{\mathrm{e}}$} \\
\hline & $5-12 \mathrm{~h}$ & 14 & 0.12 & $-0.01,0.25$ & \\
\hline & $12-18 \mathrm{~h}$ & 11 & $0.38^{\mathrm{d}}$ & $0.23,0.53$ & \\
\hline & $18-30 \mathrm{~h}$ & 9 & 0.17 & $-0.003,0.33$ & \\
\hline & Missing & 6 & & & \\
\hline
\end{tabular}

${ }^{\mathrm{a}} m=$ number of interventions

${ }^{\mathrm{b}} Q=Q$ for difference between subgroups

c The effect of the use of specific intervention techniques was investigated in symptom-oriented interventions only $(m=31)$

${ }^{\mathrm{d}} p<0.01$

e $p<0.05$

meta-analysis were categorized as low or medium quality studies, there was a lack of high quality studies. Furthermore, a range of psychosocial intervention types can be effective for PLWH, from symptom-oriented interventions such as CBT, to supportive interventions and meditation. There were no differences in effectiveness between these different intervention types, so they all seem to be helpful in improving the mental health of PLWH.

The second aim of the current meta-analysis was to study moderators of intervention effect, to determine whether important characteristics of a study or a therapy may influence the effectiveness of the treatments in question. We found that six factors may influence the effectiveness of a treatment for depression. Of these six factors, three could be classified as intervention characteristics: who provided the intervention, whether mental health was a primary focus of the intervention, and what the duration of treatment was. The other three were study characteristics: whether there was a priori screening for depression, what percentage of the participants in a study had AIDS, and what type of control group was included. Other factors, such as intervention techniques, were shown not to moderate intervention effect.

We found that, overall, psychosocial interventions had a positive effect on depression, anxiety, quality of life, and psychological well-being of PLWH. However, the pooled effect size was small, $\hat{g}=0.19$. When the pooled effect sizes of the separate outcomes were examined, it was found that the pooled effect sizes on depression and psychological well-being were the largest; smaller pooled effect sizes were found on anxiety and quality of life. Previous metaanalyses [14-16] found mostly small to moderate effect sizes of psychosocial interventions for PLWH for the outcomes depression and anxiety. The effect size on the outcome quality of life was comparable with a previous meta-analysis, which also found a small effect [16]. Furthermore, two previous meta-analyses investigated the effects of CBT and stress-management on stress (stress was included in the outcome psychological well-being in the present meta-analysis), and one of these found a moderate [14] and the other a small pooled effect size [16]. These differences in effect sizes between the current study and previous ones may be explained by a difference in the focus of the interventions included. When the moderator analysis was conducted, it was found that when mental health was the primary focus of an intervention, the effects were larger than when this was not the primary focus of an intervention. Previous meta-analyses mainly included interventions whose primary aim was to improve mental health. This important difference between the current meta- 
analysis and previous meta-analyses may explain the smaller effects in this study. In addition, the current metaanalysis included 62 studies, while most previous metaanalyses included less than half this number of studies. The inclusion criteria of this meta-analysis were also broader: various psychosocial interventions and outcomes were included. For these reasons, this meta-analysis may have more power to detect a true effect.

Furthermore, the analysis was conducted at several time points, to study the effect of interventions in both the short and the long term. It was found that the effect size was much smaller at the last time point (9 months or more after the intervention had ended), than at the earlier time points. Two previous meta-analyses $[14,18]$ about the effectiveness of CBT for PLWH with mental health problems also found that the effects were smaller on later follow-up assessments. This may indicate that the positive effects of interventions on the mental health of PLWH may wear off after a while. Booster sessions, follow-up sessions after termination of the therapy to prevent relapse, could be helpful to retain the effects. A similar finding emerged in a meta-analysis about the long-term effects of psychotherapy for depression [94]. However, only nine studies in our meta-analysis had data available on the last time point, so the results should be interpreted with caution. For future studies into psychosocial interventions for PLWH with mental health problems, we advise including a longer follow-up period to further investigate the long-term effects. In addition, future research could study the effect of booster sessions.

In the analysis on intervention types, no differences were found between the various intervention types. This is in line with previous meta-analyses about psychological therapies for depression in the general population or in people with medical disorders, which also found no differences in effectiveness between interventions such as CBT, interpersonal therapy, supportive therapy, and problem-solving therapy [94-98]. It seems that several types of interventions may be useful to improve the mental health of PLWH. It has previously been argued more generally that various forms of psychotherapy may have the same effect, because they share common factors, such as the relationship with the therapist $[99,100]$. The specific type of therapy does not seem to be that important. This corresponds with our findings. For a more thorough investigation of the differences in effect between psychosocial interventions for PLWH, it is important to design studies that compare different types of interventions. Furthermore, it would also be interesting to know more about how treatments work (mediating factors), and to compare this between different treatments. Future studies should focus on these topics.
In addition to the analysis on intervention types, we also investigated differences in effect between intervention techniques in the symptom-oriented interventions. No differences in effect were found between symptom-oriented interventions that included techniques of relaxation, CBT, stress-management, or motivational interviewing, on the one hand, and symptom-oriented interventions that did not include these techniques, on the other. This is related to the findings about intervention types and a previous metaanalysis that also did not find any differences between interventions that included or did not include stress management skills training [14].

The subgroup analyses indicated that there were several moderators that influenced the effects of interventions on depression. An important moderator was a priori screening for depressive symptoms: when studies included only participants with depressive symptoms the effect sizes were larger than when the presence of depressive symptoms did not serve as an inclusion criterion. This result seems evident: there is more to gain for PLWH with depressive symptoms than for PLWH without depressive symptoms. A previous meta-analysis also found that in studies that included PLWH with more anxiety symptoms at baseline, the participants benefited more from stressmanagement interventions [16]. On the other hand, another meta-analysis into the effectiveness of CBT for PLWH with depression and anxiety found no inclusion-related differences between studies: there was no difference between studies that restricted participation to those with depressive symptoms and studies that did not have the presence of depressive symptoms as an inclusion criterion [14]. However, in this meta-analysis the number of studies in each category was low. All in all, when an intervention is aimed at reducing depression, it seems favorable to screen PLWH a priori and only offer them the treatment if they actually suffer from depressive symptoms.

Another significant moderator was whether mental health was the primary focus of an intervention. In studies where mental health was the primary focus of the intervention, the effects were larger than in studies where mental health was not the primary focus. Again, this result seems logical: if the aim of an intervention is to reduce depression, participants will work on reducing symptoms during treatment, and it is expected that this will be effective. If the aim of an intervention is to quit smoking, for example, this will be the focus of the therapy and it is not so likely that participants' psychological symptoms will also improve.

We found that studies that had a psychologist or psychotherapist as a provider of the intervention had the largest pooled effect sizes. Studies that had other providers, e.g., counsellors, peers, or computers, all had smaller effect sizes in general. This shows that it may not be so important 
which specific therapy or technique is used to treat mental health problems in PLWH; the key element may be the provider of the intervention. Psychologists have a broad training in treating mental health problems, so they may be more experienced and more competent to help PLWH in need. This is in contrast with most other providers; they may be trained to provide the intervention, but this may not be comparable to psychologists' education and experience in mental health care. However, a previous meta-analysis about CBT for depression and anxiety in PLWH found no differences in effects between studies in which interventions were provided by a psychologist or psychiatrist and studies in which interventions were delivered by trained research staff (e.g., graduate- and doctoral-level students) [14]. Further, two meta-analyses on guided self-help [101] or guided computerized interventions [102] for depression or anxiety also did not find differences in effects between studies involving experienced providers (e.g., psychologists) and those involving less experienced providers (e.g., students). However, the moderator analysis in this metaanalysis comprised many more studies than those in the previous meta-analyses, so it has more power to detect differences. To conclude, psychologists and psychotherapists may be the most qualified providers of psychosocial treatments for PLWH with mental health problems. More research is needed to confirm this.

The duration of treatment was another important moderator in this meta-analysis. We found that studies with a treatment duration of 12-18 h had the largest effect sizes, compared to treatments of shorter or longer duration. So, it seems that therapies of average duration may be more effective than treatments of short or long duration. However, there is a trend toward designing concise treatments for mental health problems, which can be provided via the Internet and are thought to be more cost-effective. An RCT that compared the effectiveness of concise CBT with standard CBT for depression and anxiety found that they were equivalent [103]. Most previous meta-analyses that have investigated the effectiveness of psychological interventions for depression or anxiety have also found no differences between treatments with a short or long duration $[14,95,97,101,102]$. One meta-analysis about online CBT for patients with chronic somatic conditions and depression did find an effect of treatment duration, with a larger effect size for treatments with a longer duration [104]. Some of the previous meta-analyses $[14,101,102,104]$ included a small number of studies in the moderator analysis, but other meta-analyses [95, 97] included more than 100 studies. Hence, it is not yet clear whether the effectiveness of a treatment is related to its duration. When comparing short and long treatments for mental health problems, it may be useful to take the severity of the symptoms into account. People with more severe symptoms may need more sessions than people with a mild or moderate symptom severity [105]. Future studies may focus on this topic.

Contrary to expectations, the type of control group was a significant moderator in this meta-analysis. Studies that had a waiting list control group had smaller effect sizes than studies with a standard care or active control group. This is counterintuitive, since participants on a waiting list do not receive any treatment, which would lead us to expect large differences between the intervention and the control condition [106]. However, there were only five studies in the moderator analysis that used a waiting list control condition, so firm conclusions cannot be drawn. Previous meta-analyses on the effectiveness of interventions for depression and anxiety found no differences between control group types [14, 94, 102], or found that the studies that used a waiting list control condition had larger effect sizes than other control group types [95, 101, 102].

The last significant moderator was the percentage of participants in the study who had AIDS. When fewer than $40 \%$ of the participants in a study had received a diagnosis of AIDS, the effect sizes were larger than when more than $40 \%$ of the participants had AIDS. People with AIDS are generally more physically ill-they suffer from more pain and lack of energy - than people without AIDS. This physical discomfort may have a great influence on their mental well-being: i.e., they may feel more sad, worry more, or have difficulties sleeping [1]. Therefore, it may be more difficult to treat these psychological symptoms in people with AIDS. That is, the physical symptoms remain, and their influence on the mental state may hamper a successful response to treatment. Therefore, it may be important to combine medical and psychosocial treatments in people with AIDS, to try to improve or stabilize both the physical and psychological symptoms. In this situation, effective collaboration between treatment providers is crucial. It should be noted that this moderator analysis was based on only ten studies, so the results should be interpreted cautiously and may not be generalizable to other study samples.

This meta-analysis had some limitations, which will be discussed here. First, there was evidence of publication bias. This may indicate that studies with negative effect sizes were missing in the analysis. When these possible missing studies were imputed and a corrected effect size was calculated, it was smaller than the uncorrected effect size. So, it has to be concluded that the overall effect of psychosocial interventions on the mental health of PLWH is small. Second, the quality of the studies included was mostly low or medium; only $16 \%$ of the studies included was of high quality. The quality criteria regarding the concealment of allocation to conditions and the availability 
of a published study protocol, especially, were often not clearly reported in the studies included. Therefore, it is possible that some studies were classified as low or medium quality studies now, while they may have been classified as high quality studies when there would be more information in the paper concerning these criteria. Besides this, it is evident that low quality studies often did not do an intent-to-treat analysis. It would be advisable for future studies to state whether they have complied with the criteria, and that incomplete outcome data will be adequately addressed. A limitation of the instruments used to assess study quality is that when many criteria are not clearly described in a paper, a study was classified as a low quality study. However, study quality was not a significant moderator in the analysis on depression, so studies of high quality did not have larger effect sizes than studies of lower quality. Third, in some of the moderator analyses, only a few studies could be included. This is related to the fact that some studies did not report on all moderator variables. Consequently, the results of the moderator analyses with few studies may not be representative for all of the studies included, and the power is lower in these analyses. Fourth, many moderator analyses were performed in this study, and no correction for multiple testing was applied. This increases the risk of finding spurious moderator effects. Fifth, the outcomes in this meta-analysis were restricted, so the effect of psychosocial interventions on other relevant outcomes (e.g., PTSD) was not investigated. Though, a recent review [107] found two CBT-based interventions that were effective in decreasing PTSD symptoms in PLWH. Therefore, it is possible that the findings of the current meta-analysis also apply to PTSD. However, only two studies were found in the review, so more research into interventions for PTSD in PLWH is necessary. Sixth, although we searched in three databases and in the references of previous meta-analyses and reviews, it is still possible that some relevant articles were not found with this search strategy. Seventh, the moderator concerning the theory content of the intervention had two categories: theory driven or not theory-driven. For each study, it was determined to which of the categories it belonged by reading the paper. It could be argued that this is not a completely thorough approach, because an intervention may still be based on theory, despite the fact that it is not stated in the paper. Furthermore, interventions may be evidence-based, but not based on a specific theory. Or the other way around: it may be based on a theory, but it is not evidence-based. So, it is recommended for future studies to mention in the paper whether the investigated intervention was theory-driven and/or evidence-based. Last, in the moderator analysis on the effect of intervention characteristics, all interventions were investigated separately. This approach was chosen because in some studies two interventions were investigated, and the interventions did not always belong to the same category of a moderator (e.g., one intervention in a given study may have a treatment duration of $4 \mathrm{~h}$ and another a treatment duration of $8 \mathrm{~h}$ ). Therefore, six studies were represented twice in these analyses. It would be preferable to use each study just once in the analysis, but this was not possible here.

Some recommendations for future research may be derived from the results of this meta-analysis. First, future studies should focus on investigating differences between various interventions: how they work and for whom they work. Second, the long-term effects of psychosocial interventions and the effect of booster sessions should be investigated more thoroughly in the future. Third, most studies in the current meta-analysis were conducted in the USA and in Europe. Since the prevalence of HIV is high in low and middle income countries and mental health problems are common in this population, interventions to treat these problems are needed [108]. However, there are significant barriers to providing mental health services in these countries, e.g., there is a lack of trained mental health workers [109]. Therefore, it is important that these interventions are adapted to the local culture, are brief, can be provided by non-specialists, and are tailored for PLWH. It was found that interventions for PLWH in low and middle income countries were effective when they were focused on the family and integrated into community based health care [110]. More research is recommended on mental health interventions for PLWH in low and middle income countries. Fourth, there were a lot of changes in the past twenty years in the mental health care for PLWH and in study methodologies. We investigated the effects of many moderators, but there will be issues that were not addressed. For future studies, it is important to be aware of the changes in mental health care and study design. Fifth, it is likely that moderator effects were related, e.g., when there was a screening for depression in a study, this may be related to the fact that the primary focus of the intervention in the study was on mental health. These relations between moderators were not examined in the current meta-analysis, but are interesting to investigate in future meta-analyses. The most optimal result of an intervention may be obtained when the most effective characteristics will be combined in an intervention. Sixth, the moderation analysis indicated that the effect sizes were larger for studies that solely included PLWH with mental health problems and for interventions that were focused on mental health. Therefore, it is recommended in future intervention studies to restrict the inclusion to participants with mental health problems and to design interventions that are focused on improving mental health. Last, for new studies, it is important to measure and report on study and treatment characteristics, 
so that studies and interventions can be compared in metaanalyses.

To conclude, this systematic review and meta-analysis included 62 RCTs and therefore has high power. In addition, the effects of multiple intervention and study characteristics on treatment outcome were investigated. The meta-analysis found that, overall, psychosocial interventions may have a small positive effect on the mental health of PLWH. No differences in effect were found between the three intervention types, which means that symptom-oriented interventions, supportive interventions, and meditation may all be effective. A larger improvement in depression may be obtained when only participants with depressive symptoms are included in the study; when interventions are provided by psychologists; when treatment duration is $12-18 \mathrm{~h}$; and when the intervention is focused on improving mental health. Based on the results of this meta-analysis, it is important to incorporate psychosocial therapies into the care of PLWH with mental health problems.

Acknowledgements This study was supported by the Aids Fonds (File Number 2013027). We thank Anne van Woezik and Dianne van der Heide for their help in selecting the studies and extracting and coding the data.

Funding This study was funded by the Aids Fonds (file number 2013027).

\section{Compliance with Ethical Standards}

Conflict of interest All the authors declare that they have no conflict of interest.

Ethical Approval This article does not contain any studies with human participants or animals performed by any of the authors.

Open Access This article is distributed under the terms of the Creative Commons Attribution 4.0 International License (http://crea tivecommons.org/licenses/by/4.0/), which permits unrestricted use, distribution, and reproduction in any medium, provided you give appropriate credit to the original author(s) and the source, provide a link to the Creative Commons license, and indicate if changes were made.

\section{Appendix: Search Strategy}

\section{PubMed Search Term}

(hiv [mesh] OR hiv infection [mesh] OR hiv [tiab] or aids [tiab]) AND (psychotherapy [mesh] OR mental health services [mesh] OR self-care [mesh] OR self-help groups [mesh] OR telemedicine [mesh] OR therapy, computerassisted [mesh] OR psychotherap* [tiab] OR psychological therap* [tiab] OR psychological treatment* [tiab] OR psychological intervention* [tiab] OR counsel* [tiab] OR cbt [tiab] OR behavior therap* [tiab] OR behaviour therap* [tiab] OR interpersonal therap* [tiab] OR coping [tiab] OR peer support [tiab] OR social support [tiab] OR problem solving [tiab] OR stress manage* [tiab] OR self-help [tiab] OR internet therap* [tiab] OR online therap* [tiab] OR

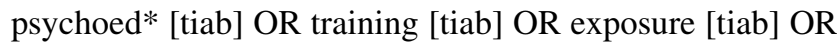
relaxation [tiab] OR mindfulness [tiab] OR reinforcement [tiab] OR risk reduction [tiab] OR commitment therap* [tiab] OR case manage* [tiab]) AND (depression [mesh] OR depress* [tiab] OR anxiety [mesh] OR anxi* [tiab] OR fear [tiab] OR quality of life [mesh] OR quality-of-life [tiab] OR well-being [tiab] OR stress, psychological [mesh] OR stress* [tiab] OR distress [tiab] OR mental health [tiab])

Filters used controlled clinical trial or randomized controlled trial, publication date from 1996/01/01, humans, English language.

\section{PsycInfo Search Term}

(DE (hiv OR aids) OR TX (hiv OR aids)) AND (DE (psychotherapy OR psychotherapeutic techniques OR mental health programs OR Counseling OR Stress management OR case management OR self management OR Telemedicine OR Computer Assisted Therapy OR Psychoeducation) OR TX (psychotherap* OR psychological-therap* OR psychological-treatment OR psychological-intervention OR counsel* OR cbt OR behavio\#r-therap* OR interpersonal-therap* OR coping OR peer-support OR social-support OR problem-solving OR stress-manage* OR self-help OR internet-therap* OR online-therap* OR psychoed* OR training OR exposure OR relaxation OR mindfulness OR reinforcement OR risk-reduction OR commitment-therap* OR case-manage*)) AND (DE (major depression OR anxiety OR stress OR distress OR quality of life OR well being) OR TX(depress* OR anxi* OR fear OR quality-of-life OR well-being OR stress* OR distress OR mental-health))

Filters used publication year 1996-2015, adulthood (18 years and older), experimental replication or treatment outcome/clinical trial or follow-up study.

\section{Embase Search Term}

(exp "Human immunodeficiency virus"/OR exp "Human immunodeficiency virus infection"/OR exp "Acquired immune deficiency syndrome"/OR hiv.tw. OR aids.tw.) AND (exp psychotherapy/or exp "mental health services"/ or exp "self care"/OR exp "self help"/OR exp teletherapy/ OR exp "computer assisted therapy"/OR psychotherap*.tw. OR psychological-therapy.tw. OR psychologicaltreatment.tw. OR psychological-intervention.tw. OR 
counsel*.tw. OR cbt.tw. OR behavio?r-therapy.tw. OR interpersonal-therapy.tw. OR coping.tw. OR peer-support.tw. OR social-support.tw. OR problem-solving.tw. OR stress-management.tw. OR self-help.tw. OR internet-therap*.tw. OR online-therap*.tw. OR psychoed*.tw. OR training.tw. OR exposure.tw. OR relaxation.tw. OR mindfulness.tw. OR reinforcement.tw. OR risk-reduction.tw. OR commitment-therap*.tw. OR case-manage*.tw.) AND (exp depression/OR depress*.tw. OR exp anxiety/OR anxi*.tw. OR exp fear/OR fear.tw. OR "quality of life"/ $\exp$ OR quality-of-life.tw. OR well-being.tw. OR exp stress/OR “mental stress"/exp OR stress*.tw. OR distress.tw. OR mental-health.tw.)

Filters used human, English language, records from Embase, randomized controlled trial or controlled clinical trial, publication year 1996-2015, article and adult (18-64 years) or aged (65+ years).

\section{References}

1. Merlin JS, Cen LY, Praestgaard A, Turner M, Obando A, Alpert $\mathrm{C}$, et al. Pain and physical and psychological symptoms in ambulatory HIV patients in the current treatment era. J Pain Symptom Manag. 2012;43(3):638-45.

2. Morrison MF, Petitto JM, Ten Have T, Gettes DR, Chiappini MS, Weber AL, et al. Depressive and anxiety disorders in women with HIV infection. Am J Psychiatry. 2002;159(5): 789-96.

3. Pence BW, Miller WC, Whetten K, Eron JJ, Gaynes BN. Prevalence of DSM-IV-defined mood, anxiety, and substance use disorders in an HIV clinic in the southeastern United States. JAIDS. 2006;42(3):298-306.

4. Nanni MG, Caruso R, Mitchell AJ, Meggiolaro E, Grassi L. Depression in HIV infected patients: a review. Curr Psychiatry Rep. 2015;17(1):11.

5. Evans DL, Charney DS, Lewis L, Golden RN, Gorman JM, Krishnan KR, et al. Mood disorders in the medically ill: scientific review and recommendations. Biol Psychiatry. 2005; 58(3): 175-89.

6. Vitiello B, Burnam MA, Bing EG, Beckman R, Shapiro MF. Use of psychotropic medications among HIV-infected patients in the United States. Am J Psychiatry. 2003;160(3):547-54.

7. Lee RS, Kochman A, Sikkema KJ. Internalized stigma among people living with HIV-AIDS. AIDS Behav. 2002;6(4):309-19.

8. Gonzalez JS, Batchelder AW, Psaros C, Safren SA. Depression and HIV/AIDS treatment nonadherence: a review and metaanalysis. J Acquir Immune Defic Syndr. 2011;58:181-7.

9. Ironson G, O'Cleirigh C, Fletcher MA, Laurenceau JP, Balbin E, Klimas N, et al. Psychosocial factors predict CD4 and viral load change in men and women with human immunodeficiency virus in the era of highly active antiretroviral treatment. Psychosom Med. 2005;67(6):1013-21.

10. Leserman J, Petitto JM, Perkins DO, Folds JD, Golden RN, Evans DL. Severe stress, depressive symptoms, and changes in lymphocyte subsets in human immunodeficiency virus-infected men-a 2-year follow-up study. Arch Gen Psychiatry. 1997; 54(3):279-85.

11. Sherbourne CD, Hays RD, Fleishman JA, Vitiello B, Magruder $\mathrm{KM}$, Bing EG, et al. Impact of psychiatric conditions on health- related quality of life in persons with HIV infection. Am J Psychiatry. 2000;157(2):248-54.

12. Brown JL, Vanable PA. Cognitive-behavioral stress management interventions for persons living with HIV: a review and critique of the literature. Ann Behav Med. 2008;35(1): 26-40.

13. Clucas C, Sibley E, Harding R, Liu L, Catalan J, Sherr L. A systematic review of Interventions for anxiety in people with HIV. Psychol Health Med. 2011;16(5):528-47.

14. Crepaz N, Passin WF, Herbst JH, Rama SM, Malow RM, Purcell DW, et al. Meta-analysis of cognitive-behavioral interventions on HIV-positive persons' mental health and immune functioning. Health Psychol. 2008;27(1):4-14.

15. Himelhoch S, Medoff DR, Oyeniyi G. Efficacy of group psychotherapy to reduce depressive symptoms among HIV-infected individuals: a systematic review and meta-analysis. AIDS Patient Care STDS. 2007;21(10):732-9.

16. Scott-Sheldon LAJ, Kalichman SC, Carey MP, Fielder RL. Stress management interventions for HIV+ adults: a metaanalysis of Randomized controlled trials, 1989-2006. Health Psychol. 2008;27(2):129-39.

17. Sherr L, Clucas C, Harding R, Sibley E, Catalan J. HIV and depression-a systematic review of interventions. Psychol Health Med. 2011;16(5):493-527.

18. Spies G, Asmal L, Seedat S. Cognitive-behavioural interventions for mood and anxiety disorders in HIV: a systematic review. J Affect Disord. 2013;150(2):171-80.

19. Landis JR, Koch GG. Measurement of observer agreement for categorical data. Biometrics. 1977;33(1):159-74.

20. Higgins JPT, Altman DG, Gotzsche PC, Juni P, Moher D, Oxman $\mathrm{AD}$, et al. The Cochrane Collaboration's tool for assessing risk of bias in randomised trials. BMJ. 2011; 343:9.

21. Chambless DL, Hollon SD. Defining empirically supported therapies. J Consult Clin Psychol. 1998;66(1):7-18.

22. Sarna A, Luchters S, Geibel S, Chersich MF, Munyao P, Kaai S, et al. Short-and long-term efficacy of modified directly observed antiretroviral treatment in Mombasa, Kenya: a randomized trial. JAIDS. 2008;48(5):611-9.

23. Brazier A, Mulkins A, Verhoef M. Evaluating a yogic breathing and meditation intervention for individuals living with HIV/ AIDS. Am J Health Promot. 2006;20(3):192-5.

24. Rao D, Nainis N, Williams L, Langner D, Eisin A, Paice J. Art therapy for relief of symptoms associated with HIV/AIDS. AIDS Care. 2009;21(1):64-9.

25. Rotheram-Borus MJ, Rice E, Comulada WS, Best K, Elia C, Peters $\mathrm{K}$, et al. Intervention outcomes among HIV-affected families over 18 months. AIDS Behav. 2012;16(5):1265-75.

26. Stein MD, Herman DS, Bishop D, Anderson BJ, Trisvan E, Lopez R, et al. A telephone-based intervention for depression in HIV patients: negative results from a randomized clinical trial. AIDS Behav. 2007;11(1):15-23.

27. Weber R, Christen L, Christen S, Tschopp S, Znoj H, Schneider $\mathrm{C}$, et al. Effect of individual cognitive behaviour intervention on adherence to antiretroviral therapy: prospective randomized trial. Antivir Ther. 2004;9(1):85-95.

28. Cohen J. A power primer. Psychol Bull. 1992;112(1):155-9.

29. Follmann D, Elliott P, Suh I, Cutler J. Variance imputation for overviews of clinical trials with continuous response. J Clin Epidemiol. 1992;45(7):769-73.

30. Hedges LV, Olkin I. Statistical methods for meta-analysis. Cambridge: Academic press; 1985.

31. Higgins JP, Thompson SG, Deeks JJ, Altman DG. Measuring inconsistency in meta-analyses. BMJ. 2003;327(7414):557-60.

32. Borenstein M, Hedges LV, Higgins JPT, Rothstein HR. Introduction to meta-analysis. Hoboken: Wiley; 2009. 
33. Egger M, Smith GD, Schneider M, Minder C. Bias in metaanalysis detected by a simple, graphical test. BMJ. 1997; 315(7109):629-34.

34. Duval S, Tweedie R. Trim and fill: a simple funnel-plot-based method of testing and adjusting for publication bias in metaanalysis. Biometrics. 2000;56(2):455-63.

35. Balfour L, Kowal J, Silverman A, Tasca GA, Angel JB, Macpherson PA, et al. A randomized controlled psycho-education intervention trial: improving psychological readiness for successful HIV medication adherence and reducing depression before initiating HAART. AIDS Care. 2006;18(7):830-8.

36. Berger S, Schad T, von Wyl V, Ehlert U, Zellweger C, Furrer H, et al. Effects of cognitive behavioral stress management on HIV1 RNA, CD4 cell counts and psychosocial parameters of HIVinfected persons. AIDS. 2008;22(6):767-75.

37. Boivin MJ, Bangirana P, Nakasujja N, Page CF, Shohet C, Givon D, et al. A year-long caregiver training program improves cognition in preschool Ugandan children with human immunodeficiency virus. J Pediatr. 2013;163(5):1409-16 e1-5.

38. Boivin MJ, Bangirana P, Nakasujja N, Page CF, Shohet C, Givon D, et al. A year-long caregiver training program to improve neurocognition in preschool Ugandan HIV-exposed children. J Dev Behav Pediatr. 2013;34(4):269-78.

39. Bormann JE, Gifford AL, Shively M, Smith TL, Redwine L, Kelly A, et al. Effects of spiritual mantram repetition on HIV outcomes: a randomized controlled trial. J Behav Med. 2006;29(4):359-76.

40. Brown JL, Vanable PA, Carey MP, Elin L. Computerized stress management training for HIV+ women: a pilot intervention study. AIDS Care. 2011;23(12):1525-32.

41. Carrico AW, Antoni MH, Duran RE, Ironson G, Penedo F, Fletcher MA, et al. Reductions in depressed mood and denial coping during cognitive behavioral stress management with HIV-positive gay men treated with HAART. Ann Behav Med. 2006;31(2): 155-64

42. Carrico AW, Chesney MA, Johnson MO, Morin SF, Neilands $\mathrm{TB}$, Remien RH, et al. Randomized controlled trial of a cognitive-behavioral intervention for HIV-positive persons: an investigation of treatment effects on psychosocial adjustment. AIDS Behav. 2009;13(3):555-63.

43. Chan I, Kong P, Leung P, Au A, Li P, Chung R, et al. Cognitivebehavioral group program for Chinese heterosexual HIV-infected men in Hong Kong. Patient Educ Couns. 2005;56(1): 78-84.

44. Chang BH, Boehmer U, Zhao Y, Sommers E. The combined effect of relaxation response and acupuncture on quality of life in patients with HIV: a pilot study. J Altern Complement Med. 2007;13(8):807-15.

45. Chhatre S, Metzger DS, Frank I, Boyer J, Thompson E, Nidich $S$, et al. Effects of behavioral stress reduction transcendental meditation intervention in persons with HIV. AIDS Care. 2013;25(10):1291-7.

46. Cote JK, Pepler C. A randomized trial of a cognitive coping intervention for acutely ill HIV-positive men. Nurs Res. 2002;51(4):237-44

47. Duncan LG, Moskowitz JT, Neilands TB, Dilworth SE, Hecht FM, Johnson MO. Mindfulness-based stress reduction for HIV treatment side effects: a randomized, wait-list controlled trial. J Pain Symptom Manag. 2012;43(2):161-71.

48. Eller LS, Kirksey KM, Nicholas PK, Corless IB, Holzemer WL, Wantland DJ, et al. A randomized controlled trial of an HIV/ AIDS symptom management manual for depressive symptoms. AIDS Care. 2013;25(4):391-9.

49. Erlen JA, Mellors MP, Sereika SM, Cook C. The use of life review to enhance quality of life of people living with AIDS: a feasibility study. Qual Life Res. 2001;10(5):453-64.
50. Fife BL, Scott LL, Fineberg NS, Zwickl BE. Promoting adaptive coping by persons with HIV disease: evaluation of a patient/partner intervention model. J Assoc Nurses AIDS Care. 2008;19(1):75-84.

51. Gayner B, Esplen MJ, DeRoche P, Wong J, Bishop S, Kavanagh $\mathrm{L}$, et al. A randomized controlled trial of mindfulness-based stress reduction to manage affective symptoms and improve quality of life in gay men living with HIV. J Behav Med. 2012;35(3):272-85.

52. Heckman TG, Carlson B. A randomized clinical trial of two telephone-delivered, mental health interventions for HIV-infected persons in rural areas of the United States. AIDS Behav. 2007;11(1):5-14.

53. Heckman TG, Sikkema KJ, Hansen N, Kochman A, Heh V, Neufeld S, et al. A randomized clinical trial of a coping improvement group intervention for HIV-infected older adults. J Behav Med. 2011;34(2):102-11.

54. Heckman TG, Heckman BD, Anderson T, Lovejoy TI, Mohr D, Sutton M, et al. Supportive-expressive and coping group teletherapies for HIV-infected older adults: a randomized clinical trial. AIDS Behav. 2013;17(9):3034-44.

55. Hersch RK, Cook RF, Billings DW, Kaplan S, Murray D, Safren $\mathrm{S}$, et al. Test of a web-based program to improve adherence to HIV medications. AIDS Behav. 2013;17(9):2963-76.

56. Ironson G, O’Cleirigh C, Leserman J, Stuetzle R, Fordiani J, Fletcher $\mathrm{M}$, et al. Gender-specific effects of an augmented written emotional disclosure intervention on posttraumatic, depressive, and HIV-disease-related outcomes: a randomized, controlled trial. J Consult Clin Psychol. 2013;81(2):284-98.

57. Jensen SE, Pereira DB, Whitehead N, Buscher I, McCalla J, Andrasik M, et al. Cognitive-behavioral stress management and psychological well-being in HIV+ racial/ethnic minority women with human papillomavirus. Health Psychol. 2013; 32(2):227-30.

58. Kaaya SF, Blander J, Antelman G, Cyprian F, Emmons KM, Matsumoto K, et al. Randomized controlled trial evaluating the effect of an interactive group counseling intervention for HIVpositive women on prenatal depression and disclosure of HIV status. AIDS Care. 2013;25(7):854-62.

59. Kalichman SC. The other side of the healthy relationships intervention: mental health outcomes and correlates of sexual risk behavior change. AIDS Educ Prev. 2005;17(1 Suppl A):66-75.

60. Klein CH, Lomonaco CG, Pavlescak R, Card JJ. WiLLOW: reaching HIV-positive African-American women through a computer-delivered intervention. AIDS Behav. 2013;17(9): 3013-23.

61. Kraaij V, van Emmerik A, Garnefski N, Schroevers MJ, Lo-FoWong D, van Empelen P, et al. Effects of a cognitive behavioral self-help program and a computerized structured writing intervention on depressed mood for HIV-infected people: a pilot randomized controlled trial. Patient Educ Couns. 2010;80(2): 200-4.

62. Lechner SC, Antoni MH, Lydston D, LaPerriere A, Ishii M, Devieux $\mathrm{J}$, et al. Cognitive-behavioral interventions improve quality of life in women with AIDS. J Psychosom Res. 2003;54(3):253-61.

63. Li L, Lee S-J, Jiraphongsa C, Khumtong S, Iamsirithaworn S, Thammawijaya $\mathrm{P}$, et al. Improving the health and mental health of people living HIV/AIDS: 12-month assessment of a behavioral intervention in Thailand. Am J Public Health. 2010; 100(12):2418-25.

64. Lovejoy TI. Telephone-delivered motivational interviewing targeting sexual risk behavior reduces depression, anxiety, and stress in HIV-positive older adults. Ann Behav Med. 2012; 44(3):416-21. 
65. McCain NL, Gray DP, Elswick RK, Robins JW, Tuck I, Walter $\mathrm{JM}$, et al. A randomized clinical trial of alternative stress management interventions in persons with HIV infection. J Consult Clin Psychol. 2008;76(3):431-41.

66. Miles MS, Holditch-Davis D, Eron J, Black BP, Pedersen C, Harris DA. An HIV self-care symptom management intervention for African American mothers. Nurs Res. 2003;52(6): 350-60.

67. Miller DK, Chibnall JT, Videen SD, Duckro PN. Supportiveaffective group experience for persons with life-threatening illness: reducing spiritual, psychological, and death-related distress in dying patients. J Palliat Med. 2005;8(2):333-43.

68. Mitrani VB, McCabe BE, Burns MJ, Feaster DJ. Family mechanisms of structural ecosystems therapy for HIV-seropositive women in drug recovery. Health Psychol. 2012;31(5): 591-600.

69. Murphy DA, Lu MC, Martin D, Hoffman D, Marelich WD. Results of a pilot intervention trial to improve antiretroviral adherence among HIV-positive patients. J Assoc Nurses AIDS Care. 2002;13(6):57-69.

70. Murphy DA, Armistead L, Marelich WD, Payne DL, Herbeck DM. Pilot trial of a disclosure intervention for HIV + mothers: the TRACK program. J Consult Clin Psychol. 2011;79(2): 203-14.

71. O'Leary A, Hoff CC, Purcell DW, Gomez CA, Parsons JT, Hardnett F, et al. What happened in the SUMIT trial? Mediation and behavior change. AIDS. 2005;19(1):S111-21.

72. Olley BO. Improving well-being through psycho-education among voluntary counseling and testing seekers in Nigeria: a controlled outcome study. AIDS Care. 2006;18(8):1025-31.

73. Pacella ML, Armelie A, Boarts J, Wagner G, Jones T, Feeny N, et al. The impact of prolonged exposure on PTSD symptoms and associated psychopathology in people living with HIV: a randomized test of concept. AIDS Behav. 2012;16(5):1327-40.

74. Peltzer K, Ramlagan S, Jones D, Weiss SM, Fomundam H, Chanetsa L. Efficacy of a lay health worker led group antiretroviral medication adherence training among non-adherent HIV-positive patients in KwaZulu-Natal, South Africa: results from a randomized trial. SAHARA J. 2012;9(4):218-26.

75. Petersen I, Hanass Hancock J, Bhana A, Govender K. A groupbased counselling intervention for depression comorbid with HIV/AIDS using a task shifting approach in South Africa: a randomized controlled pilot study. J Affect Disord. 2014;158: 78-84.

76. Ransom D, Heckman TG, Anderson T, Garske J, Holroyd K, Basta T. Telephone-delivered, interpersonal psychotherapy for HIV-infected rural persons with depression: a pilot trial. Psychiatr Serv. 2008;59(8):871-7.

77. Safren SA, O'Cleirigh C, Tan JY, Raminani SR, Reilly LC, Otto MW, et al. A randomized controlled trial of cognitive behavioral therapy for adherence and depression (CBT-AD) in HIV-infected individuals. Health Psychol. 2009;28(1): $1-10$.

78. Safren SA, O'Cleirigh CM, Bullis JR, Otto MW, Stein MD, Pollack MH. Cognitive behavioral therapy for adherence and depression (CBT-AD) in HIV-infected injection drug users: a randomized controlled trial. J Consult Clin Psychol. 2012; 80(3):404-15.

79. SeyedAlinaghi S, Jam S, Foroughi M, Imani A, Mohraz M, Djavid GE, et al. Randomized controlled trial of mindfulnessbased stress reduction delivered to human immunodeficiency virus-positive patients in Iran: effects on CD4(+) T lymphocyte count and medical and psychological symptoms. Psychosom Med. 2012;74(6):620-7.

80. Shuter J, Morales DA, Considine-Dunn SE, An LC, Stanton CA. Feasibility and preliminary efficacy of a web-based smoking cessation intervention for HIV-infected smokers: a randomized controlled trial. J Acquir Immune Defic Syndr. 2014;67(1): $59-66$.

81. Hansen N, Tarakeshwar N, Ghebremichael M, Zhang H, Kochman A, Sikkema K. Longitudinal effects of coping on outcome in a randomized controlled trial of a group intervention for HIV-positive adults with AIDS-related bereavement. Death Stud. 2006;30(7):609-36.

82. Sikkema KJ, Hansen NB, Ghebremichael M, Kochman A, Tarakeshwar N, Meade CS, et al. A randomized controlled trial of a coping group intervention for adults with HIV who are AIDS bereaved: longitudinal effects on grief. Health Psychol. 2006;25(5):563-70.

83. Sikkema KJ, Hansen NB, Kochman A, Tate DC, Difranceisco W. Outcomes from a randomized controlled trial of a group intervention for HIV positive men and women coping with AIDS-related loss and bereavement. Death Stud. 2004;28(3): 187-209.

84. Sikkema KJ, Hansen NB, Meade CS, Kochman A, Lee RS. Improvements in health-related quality of life following a group intervention for coping with AIDS-bereavement among HIVinfected men and women. Qual Life Res. 2005;14(4):991-1005.

85. Simoni JM, Pantalone DW, Plummer MD, Huang B. A randomized controlled trial of a peer support intervention targeting antiretroviral medication adherence and depressive symptomatology in HIV-positive men and women. Health Psychol. 2007;26(4):488-95.

86. Simoni JM, Wiebe JS, Sauceda JA, Huh D, Sanchez G, Longoria $\mathrm{V}$, et al. A preliminary RCT of CBT-AD for adherence and depression among HIV-positive Latinos on the U.S.-Mexico border: the Nuevo Dia study. AIDS Behav. 2013;17(8): 2816-29.

87. Szapocznik J, Feaster DJ, Mitrani VB, Prado G, Smith L, Robinson-Batista C, et al. Structural ecosystems therapy for HIV-seropositive African American women: effects on psychological distress, family hassles, and family support. J Consult Clin Psychol. 2004;72(2):288-303.

88. Van Tam V, Larsson M, Pharris A, Diedrichs B, Nguyen HP, Nguyen CTK, et al. Peer support and improved quality of life among persons living with HIV on antiretroviral treatment: a randomised controlled trial from north-eastern Vietnam. Health Qual Life Outcomes. 2012;10:53.

89. Vidrine DJ, Arduino RC, Gritz ER. Impact of a cell phone intervention on mediating mechanisms of smoking cessation in individuals living with HIV/AIDS. Nicotine Tob Res. 2006;8(Suppl 1):S103-8.

90. Webel AR. Testing a peer-based symptom management intervention for women living with HIV/AIDS. AIDS Care. 2010;22(9):1029-40.

91. Williams AL, Selwyn PA, Liberti L, Molde S, Njike VY, McCorkle R, et al. A randomized controlled trial of meditation and massage effects on quality of life in people with late-stage disease: a pilot study. J Palliat Med. 2005;8(5):939-52.

92. Williams JK, Wyatt GE, Rivkin I, Ramamurthi HC, Li XM, Liu HH. Risk reduction for HIV-positive African American and Latino men with histories of childhood sexual abuse. Arch Sex Behav. 2008;37(5):763-72.

93. Williams JK, Glover DA, Wyatt GE, Kisler K, Liu H, Zhang M. A sexual risk and stress reduction intervention designed for HIV-positive bisexual African American men with childhood sexual abuse histories. Am J Public Health. 2013;103(8): 1476-84.

94. Karyotaki E, Smit Y, de Beurs DP, Henningsen KH, Robays J, Huibers $\mathrm{MJH}$, et al. The long-term efficacy of acute-phase psychotherapy for depression: a meta-analysis of randomized trials. Depress Anxiety. 2016;33(5):370-83. 
95. Cuijpers P, Berking M, Andersson G, Quigley L, Kleiboer A, Dobson KS. A meta-analysis of cognitive-behavioural therapy for adult depression, alone and in comparison with other treatments. Can J Psychiatry. 2013;58(7):376-85.

96. Cuijpers P, Geraedts AS, van Oppen P, Andersson G, Markowitz JC, van Straten A. Interpersonal psychotherapy for depression: a meta-analysis. Am J Psychiatry. 2011;168:581-92.

97. Cuijpers P, van Straten A, Bohlmeijer E, Hollon S, Andersson G. The effects of psychotherapy for adult depression are overestimated: a meta-analysis of study quality and effect size. Psychol Med. 2010;40(02):211-23.

98. van Straten A, Geraedts A, Verdonck-de Leeuw I, Andersson G, Cuijpers P. Psychological treatment of depressive symptoms in patients with medical disorders: a meta-analysis. J Psychosom Res. 2010;69(1):23-32.

99. Luborsky L, Rosenthal R, Diguer L, Andrusyna TP, Berman JS, Levitt JT, et al. The dodo bird verdict is alive and well-mostly. Clin Psychol Sci Pract. 2002;9(1):2-12.

100. Rosenzweig S. Some implicit common factors in diverse methods of psychotherapy. Am J Orthopsychiatr. 1936;6(3):412.

101. Gellatly J, Bower P, Hennessy S, Richards D, Gilbody S, Lovell $\mathrm{K}$. What makes self-help interventions effective in the management of depressive symptoms? Meta-analysis and meta-regression. Psychol Med. 2007;37(9):1217-28.

102. Newby JM, Twomey C, Li SSY, Andrews G. Transdiagnostic computerised cognitive behavioural therapy depression and anxiety: a systematic review and meta-analysis. J Affect Disord. 2016;199:30-41.

103. Meuldijk D, Carlier I, van Vliet I, van Veen T, Wolterbeek R, van Hemert A, et al. The clinical effectiveness of concise cognitive behavioral therapy with or without pharmacotherapy for depressive and anxiety disorders; a pragmatic randomized controlled equivalence trial in clinical practice. Contemp Clin Trials. 2016;47:131-8.

104. van Beugen S, Ferwerda M, Hoeve D, Rovers MM, Spillekomvan Koulil S, van Middendorp H, et al. Internet-based cognitive behavioral therapy for patients with chronic somatic conditions: a meta-analytic review. J Med Internet Res. 2014;16(3):e88.

105. Shapiro DA, Barkham M, Rees A, Hardy GE, Reynolds S, Startup M. Effects of treatment duration and severity of depression on the effectiveness of cognitive-behavioral and psychodynamic-interpersonal psychotherapy. J Consult Clin Psychol. 1994;62(3):522.

106. Mohr DC, Spring B, Freedland KE, Beckner V, Arean P, Hollon $\mathrm{SD}$, et al. The selection and design of control conditions for randomized controlled trials of psychological interventions. Psychother Psychosom. 2009;78(5):275-84.

107. Applebaum AJ, Bedoya CA, Hendriksen ES, Wilkinson JL, Safren SA, O'cleirigh C. Future directions for interventions targeting PTSD in HIV-infected adults. J Assoc Nurses AIDS Care. 2015;26(2):127-38.

108. Chibanda D, Benjamin L, Weiss HA, Abas M. Mental, neurological, and substance use disorders in people living with HIV/ AIDS in low-and middle-income countries. JAIDS. 2014; 67:S54-67.

109. Saraceno B, van Ommeren M, Batniji R, Cohen A, Gureje O, Mahoney $\mathrm{J}$, et al. Barriers to improvement of mental health services in low-income and middle-income countries. Lancet. 2007;370(9593):1164-74.

110. Sikkema KJ, Dennis AC, Watt MH, Choi KW, Yemeke TT, Joska JA. Improving mental health among people living with HIV: a review of intervention trials in low-and middle-income countries. Glob Mental Health. 2015;2:e19. 